\title{
Linear and Nonlinear Nanophotonic Devices Based on Silicon-on-Insulator Wire Waveguides
}

\author{
Pieter Dumon*, Gino Priem, Luis Romeu Nunes ${ }^{1}$, Wim Bogaerts, Dries Van Thourhout, Peter Bienstman, \\ Tak Keung Liang ${ }^{1}$, Masahiro TsuChiYA ${ }^{1}$, Patrick JAENEN ${ }^{2}$, Stephan BeCKX ${ }^{2}$, Johan Wouters ${ }^{2}$ and Roel BAets \\ Department of Information Technology-IMEC, Ghent University, Sint-Pietersnieuwstraat 41, 9000 Gent, Belgium \\ ${ }^{1}$ National Institute of Information and Communications Technology (NICT), Tokyo 100-0004, Japan \\ ${ }^{2}$ Interuniversity Micro-Electronics Center (IMEC), Leuven, Belgium
}

(Received February 28, 2006; accepted March 28, 2006; published online August 22, 2006)

We review the basic linear and nonlinear properties of silicon-on-insulator photonic wire waveguides and their application to nanophotonic circuits. We give an overview of the performance and issues of basic circuit elements such as couplers and intersections and review the achievements in linear wavelength-selective elements, as well as nonlinear applications of wires and resonators for high-speed signal processing. [DOI: 10.1143/JJAP.45.6589]

KEYWORDS: photonic wire, silicon-on-insulator, arrayed waveguide grating, ring resonator

\section{Introduction}

Submicron silicon wire (strip) waveguides offer a very attractive path towards a much larger scale integration than today's commercially available photonic integrated circuits. With their high index contrast, compact splitters and couplers and micron-sized bend radii can be realized, and passive linear functions such as wavelength division multiplexing (WDM) filters become possible within a very limited footprint. The strong field confinement enables the exploration and development of compact nonlinear functions for high-speed all-optical signal processing at acceptable power levels. Additionally, one has the possibility to use complementary metal-oxide semiconductor (CMOS) based processing and its mature and well established technology and huge knowledge base.

In this paper we give an overview of the advances in passive linear and nonlinear functions in silicon-on-insulator (SOI), with focus on our results obtained with wires fabricated by deep UV lithography. In $\S 2$ and $\S 3$, we will give an overview of the linear and nonlinear properties of SOI wires. Section 4 is a brief overview and comparison of fabrication methods for SOI nanophotonics and fiber coupling methods. Then after discussing linear circuits and some basic circuit elements in $\$ 5$, linear wavelength filter applications are reviewed in $\S 6$ and nonlinear applications in $\$ 7$.

In this paper, we restrict ourselves to passive functions, with focus on applications in telecommunication. Active photonic functions in silicon-wire like devices have seen a great rise of interest over the last years, and devices such as high-speed modulators have been demonstrated. ${ }^{1)}$ However, the possible application fields of passive SOI nanophotonic technology are diverse and extend into short distance communication such as interconnects and even environmental and biological sensors. Ultimately, passive and active devices are to be combined in order to gain full flexibility and support numerous applications.

\section{Linear Properties}

The basic waveguide structure considered is a silicon strip on top of an oxide cladding resting on a silicon substrate. Optionally, a top cladding can be considered, such as oxide

*E-mail address: Pieter.Dumon@intec.UGent.be for passivation or symmetry reasons.

The crystalline $\mathrm{Si}$ core layer and amorphous $\mathrm{SiO}_{2}$ cladding have a refractive index of 3.48 and 1.44 respectively, around $1550 \mathrm{~nm}$ and at room temperature. ${ }^{3)}$ Absorption in crystalline $\mathrm{Si}$ is neglible in the telecom wavelength range. At the $\mathrm{Si}$-air or $\mathrm{Si}$-oxide interface, non-negligible absorption may arise, depending on the fabrication process. ${ }^{4)}$

The wire supports transverse electric (TE) like and transverse magnetic (TM) like modes. Figures 1(a) and 1(b) show dispersion diagrams in wires with oxide and air topcladding respectively, for a $220 \mathrm{~nm}$ thick Si core. At $1550 \mathrm{~nm}$ wavelength, the asymmetric wire has single TEand TM-like modes up to $600 \mathrm{~nm}$ width. However, with an oxide topcladding, the single mode width is reduced to $430 \mathrm{~nm}$, even though the index contrast is slightly reduced. For a $400 \mathrm{~nm}$ wide wire, the wire is single mode upwards of $1500 \mathrm{~nm}$ wavelength. In the asymmetric case, one can see that the TE- and TM-like modes interact, making these modes completely hybrid at the anticrossing point.

The effective index of the TE ground mode in a $500 \times$ $220 \mathrm{~nm}^{2}$. waveguide is 2.44 at $1550 \mathrm{~nm}$, while the group index is 4.2 , which can be important when calculating the latency of an optical link.

The dispersion of the TE-like ground mode, including waveguide dispersion and a small contribution from material dispersion, is shown in Fig. 2. It was calculated at $1550 \mathrm{~nm}$ wavelength as a function of waveguide width. In the width range for single mode operation, the dispersion is positive and reaches a maximum value of $580 \mathrm{ps} \mathrm{nm}^{-1} \mathrm{~km}^{-1}$. This is 30 times higher than the dispersion in single mode fibers, but still only gives a propagation length limit of around $2 \mathrm{~km}$ at $10 \mathrm{GHz}$ and $140 \mathrm{~m}$ at $40 \mathrm{GHz}$. Only at several $\mathrm{THz}$, the maximum propagation length is of the order of $\mathrm{cm}$. Interesting features are the two zero dispersion points, at 410 and $655 \mathrm{~nm}$ width. The inset of Fig. 2 shows the dispersion over a larger width range.

\subsection{Losses}

Propagation losses have been reduced over the past years to a few $\mathrm{dB} / \mathrm{cm}$ by several groups. An overview is given in Table I. IMEC demonstrated $500 \times 220 \mathrm{~nm}^{2}$ wires with 2.4 $\mathrm{dB} / \mathrm{cm}$ propagation loss. These losses are still much higher than in silica waveguides or even large SOI rib waveguides, but as the minimum bend radius is reduced by orders of magnitude too, the higher propagation loss does not give a 


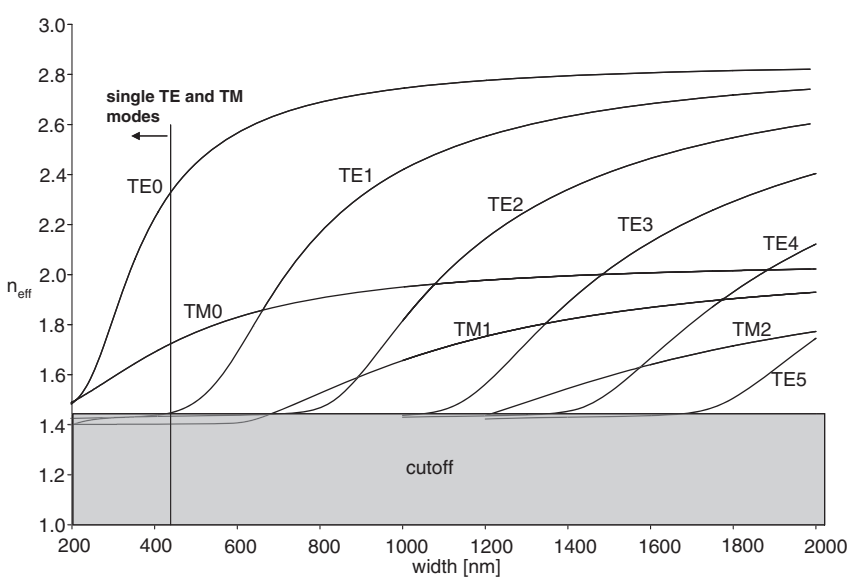

(a)

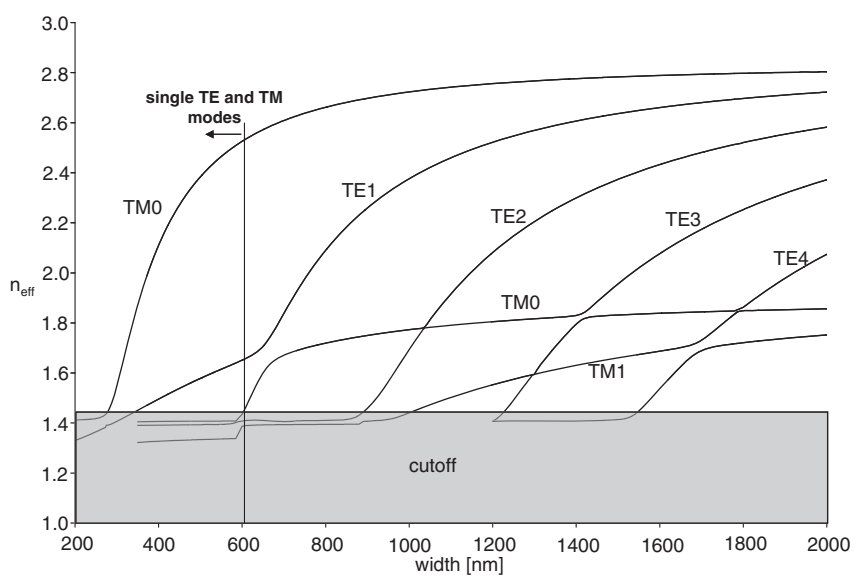

(b)

Fig. 1. Dispersion diagrams for a wires at $1550 \mathrm{~nm}$ wavelength: (a) oxide cladding and (b) oxide bottom and air topcladding.

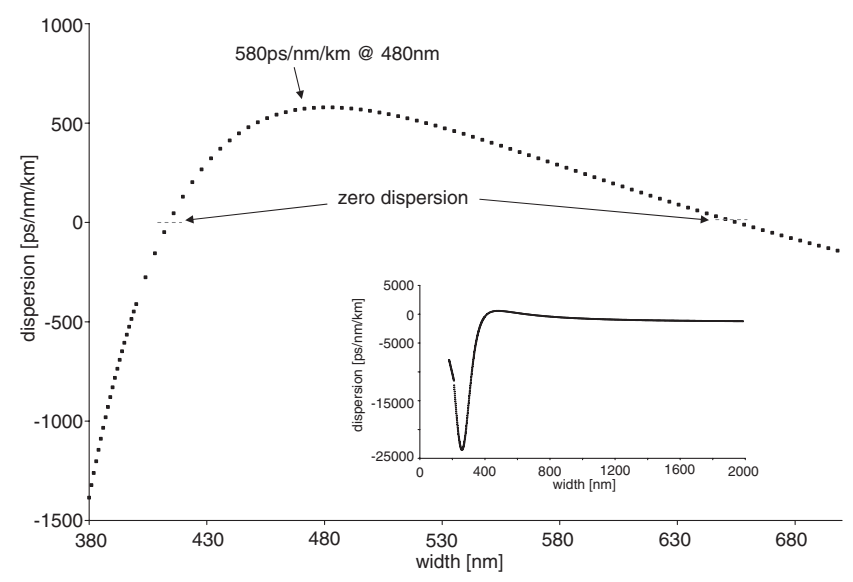

Fig. 2. Dispersion of the TE-like ground mode in a wire with $220 \mathrm{~nm} \mathrm{Si}$ thickness and oxide cladding.

higher loss per component however. In complex integrated circuits with many components and long total interconnection lengths, this picture changes. Therefore, losses need to be reduced further.

In SOI wires, propagation loss results from scattering due to sidewall roughness, substrate leakage and absorption at imperfect material interfaces. Theoretical calculations of propagation losses due to scattering at sidewall roughness
Table I. Waveguide losses.

\begin{tabular}{|c|c|c|}
\hline Affiliation & $\begin{array}{l}\text { Cross-section } \\
\left(\mathrm{nm}^{2}\right)\end{array}$ & $\begin{array}{c}\text { Loss } \\
(\mathrm{dB} / \mathrm{cm})\end{array}$ \\
\hline IMEC $^{5)}$ & $500 \times 220$ & 2.4 \\
\hline $\mathrm{IBM}^{6)}$ & $445 \times 220$ & 3.6 \\
\hline Cornell $^{27)}$ & $470 \times 270$ & 5.0 \\
\hline $\mathrm{NTT}^{10,58)}$ & $400 \times 200$ & 2.4 \\
\hline Yokohama ${ }^{13,35)}$ & $400 \times 320$ & 105.0 \\
\hline $\mathrm{MIT}^{25)}$ & $630 \times 220$ & 3.4 \\
\hline \multirow[t]{2}{*}{$\mathrm{LETI}^{24)}$} & $300 \times 300$ & 15.0 \\
\hline & $500 \times 200$ & 5.0 \\
\hline Columbia $^{14)}$ & $600 \times 260$ & 110.0 \\
\hline $\mathrm{NEC}^{34)}$ & $300 \times 300$ & 18.9 \\
\hline Aachen $/ \mathrm{AMO}^{53)}$ & $500 \times 450$ & 1.9 (in bend) \\
\hline
\end{tabular}

have been performed in full the fully three dimensional (3D) case only very recently. ${ }^{7)}$ The authors show that previous two dimensional (2D) calculations can seriously overestimate propagation losses, as the losses depend very much on the waveguide cross-section. The calculations show that for Si wires with a $220 \mathrm{~nm}$ core height, embedded in silica, the TM-like mode suffers a scattering loss which is an order of magnitude smaller than that for the TE-like mode. However, measurements show that the TE-like mode has lower propagation loss than the TM-like mode. Part of this can be explained by the higher substrate leakage due to the lower confinement for the TM mode, and possibly by a difference in surface state absorption, which might form an important contribution to the total propagation losses. ${ }^{4)}$

One of the major reasons for using high lateral and vertical index contrast is the small bend radius that can be used. We measured the excess loss for a $90^{\circ}$ bend to be smaller than $0.004 \mathrm{~dB}$ for $5 \mu \mathrm{m}$ radius and still smaller than $0.1 \mathrm{~dB}$ for $1 \mu \mathrm{m}$ radius, with spiral-like structures containing up to 550 bends for an accurate measurement. ${ }^{8)}$ Vlasov et $a l .{ }^{6)}$ demonstrated $0.086 \mathrm{~dB}$ for $1 \mu \mathrm{m}$ radius and 0.013 $\mathrm{dB}$ for $2 \mu \mathrm{m}$ radius, with structures containing 10 and 20 bends. Sakai et al. ${ }^{9)}$ experimentally found losses smaller than $0.5 \mathrm{~dB}$ for all radii. Tsuchizawa et al. ${ }^{10)}$ measured 0.15 $\mathrm{dB}$ excess loss for a $2 \mu \mathrm{m}$ radius $90^{\circ}$ bend in a $400 \times$ $200 \mathrm{~nm}^{2}$ core.

\subsection{Polarization}

One can see from Fig. 1 that the TE- and TM-like modes have very different properties in a $220 \times 500 \mathrm{~nm}^{2}$ wire. This is due to the geometrical asymmetry of the core and the different boundary conditions for each modes. Clearly, polarization independent circuits are impossible with waveguide dimensions like these. It is possible to overcome this problem by using a square waveguide core when the cladding is symmetric. However, even a slight core symmetry deviation will again lead to considerable polarization dependency, and due to the different boundary conditions for the field, the polarization dependent loss due to scattering, absorption or substrate leakage will differ.

Another problem is the polarization conversion in small bends. This was studied by Sakai et al., ${ }^{9}{ }^{9}$ reporting a simulated $-25 \mathrm{~dB}$ polarization crosstalk for a $1 \mu \mathrm{m}$ radius $90^{\circ}$ bend. However, actual measurements showed a crosstalk of up to $-10 \mathrm{~dB}$, which may be explained by a $5^{\circ}$ sidewall 

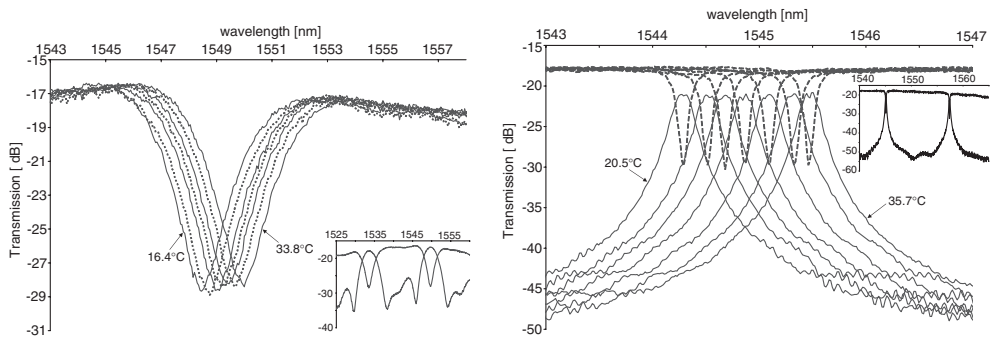

\begin{tabular}{|c|c|c|c|}
\hline device & $\begin{array}{c}\text { wire width } \\
{[\mathrm{nm}]}\end{array}$ & $\begin{array}{c}\text { band center } \\
{[\mathrm{nm}]}\end{array}$ & $\begin{array}{c}\text { shift } \\
{[\mathrm{pm} / \mathrm{K}]}\end{array}$ \\
\hline awg & 600 & 1532.5 & 69.2 \\
lattice & 560 & 1533 & 81.4 \\
& & 1550 & 89.1 \\
ring & 460 & 1552 & 83.4 \\
ring & 470 & 1540 & 85.4 \\
& & 1551 & 88.2 \\
ring & 550 & 1545 & 80.4 \\
ring & 560 & 1538 & 82.2 \\
& & 1555 & 84.4 \\
ring & 565 & 1553 & 77.0 \\
\hline
\end{tabular}

Fig. 3. Transmission measurements of filters at different temperatures and the resulting shifts for different filter band centers and isolated wire widths: (a) lattice filter, (b) ring resonators, and (c) shifts. tilt. Scattering loss can also play a role there. Additionally, the authors found a great dependency of the crosstalk on the bend pattern.

Polarization dependency is currently one of the major problems associated with SOI wire circuits. In SOI rib waveguides with large cross-sections, the problem is smaller and may be resolved, for instance using stress engineering. ${ }^{2)}$ However, SOI wires still offer a much higher integration scale and possibilities for nonlinear applications. Similar techniques may possibly be used to obtain polarization independent SOI wires with near-square cores. However, using polarization diversity may be a better approach. This can be achieved by coupling the two orthogonal fiber polarizations into separate waveguides and duplicating the circuit. With 2D grating couplers, the circuit needs to be designed for TE only (\$4.1).

\subsection{Temperature dependence}

The temperature dependence of the refractive index $\beta(T)=1 / n \cdot d n / d T$ of $\mathrm{Si}$ is high, at $1.79 \times 10^{-4} / \mathrm{K}$ at $1530 \mathrm{~nm}$ wavelength around room temperature. ${ }^{11)}$ For $\mathrm{SiO}_{2}$, $\beta$ is an order of magnitude lower (typically $1.2 \times 10^{-5} / \mathrm{K}$ ). The thermal expansion of $\mathrm{Si}$ is $2.58 \times 10^{-6} / \mathrm{K}$ around room temperature for $1530 \mathrm{~nm}$ wavelength. ${ }^{11)}$ The expansion of the waveguide cross-section is therefore negligible, although stress changes may have an impact on propagation properties. In the propagation direction, the thermal expansion can give rise to very small but noticeable spectral changes when considering wavelength-dependent structures. The thermal expansion of $\mathrm{SiO}_{2}$ is an order of magnitude smaller than that of $\mathrm{Si}$. Therefore, stress in the structures will change with temperature and can have a small influence on refractive index. From the refractive index change and maximal thermal expansion, one can easily calculate the variation of the effective index with temperature, and derive the wavelength shift of wavelength selective functions. One has to take care to include the wavelength dispersion of the effective index in the calculation, as this influences the wavelength shift greatly. For filters based on SOI wires with a $220 \mathrm{~nm}$ Si thickness, we calculate a wavelength shift of 70 to $80 \mathrm{pm} / \mathrm{K}$ depending on the waveguide width. Of course the exact shift depends on the details of the filter design. We confirmed this by measurements of ring resonators, arrayed waveguide gratings and lattice filters. Figure 3 shows transmission measurements of a ring resonator and a lattice filter at different temperatures. The measured shifts are very linear. The resulting wavelength shift for a number of different filter types and orders is shown in Fig. 8. The absence of a clear correspondence between waveguide width and shift is due to the perturbation of the wires by other structures, due to fabrication variations and measurement error. However, the measured shifts are in the 70 to $90 \mathrm{pm} / \mathrm{K}$ range, corresponding well with the calculated shift.

Baehr-Jones et al. ${ }^{12)}$ calculated and measured the temperature dependent shift in ring resonators using less confining SOI wires. In these wires, the effective index changes less with Si refractive index than in our wires, resulting in a shift of around $50 \mathrm{pm} / \mathrm{K}$.

The values reported here are an order of magnitude higher than those for silica based waveguides. Therefore SOI filters will be very sensitive to temperature changes. However, this can be used in order to tune devices, as has been done already by a number of researchers. ${ }^{14,15)}$

\section{Nonlinear Properties}

Due to its inversion symmetry, crystalline silicon does not exhibit second-order nonlinear effects. Therefore, the first type of nonlinear polarization $P$ at the frequency $\omega_{\sigma}$ that can occur, is of third-order:

$$
\mathbf{P}^{(3)}(\omega)=K \epsilon_{0} \chi^{(3)}\left(-\omega_{\sigma} ; \omega_{1}, \omega_{2}, \omega_{3}\right) \mathbf{E}_{\omega_{1}} \mathbf{E}_{\omega_{2}} \mathbf{E}_{\omega_{3}}
$$

with $\omega_{\sigma}=\omega_{1}+\omega_{2}+\omega_{3}, \epsilon_{0}$ the free-space permittivity, $\mathbf{E}_{\omega_{i}}$ the electric field component with frequency $\omega_{i}$ and $K$ a constant depending on $\omega_{1}, \omega_{2}$, and $\omega_{3}$. The exact origin of 
the nonlinear response is contained in the explicit expression of the third-order susceptibility $\chi^{(3)}\left(-\omega_{\sigma} ; \omega_{1}, \omega_{2}, \omega_{3}\right)$. In this work, special attention will be paid to nonresonant, electronic nonlinear effects, which show great potential for ultrafast signal processing, together with their secondary effects. In particular, we will not discuss in detail the Raman effect and its applications [stimulated raman scattering (SRS), coherent anti-Stokes raman scattering (CARS), lasing, ...], which in silicon is a dominantly resonant effect with a bandwidth of about $100 \mathrm{GHz}$. A more detailed description of this large and vastly growing research domain in both wire and rib waveguides can among others be found in refs. 55 and 56.

\subsection{Nonresonant, electronic effects}

In the presence of a single input signal with frequency $\omega$, two nonlinear polarizations are obtained:

$$
\begin{aligned}
\mathbf{P}_{\omega} & =\epsilon_{0} \frac{3}{4} \chi^{(3)}(-\omega ; \omega,-\omega, \omega)\left|E_{\omega}\right|^{2} \mathbf{E}_{\omega} \\
\mathbf{P}_{3 \omega} & =\epsilon_{0} \frac{1}{4} \chi^{(3)}(-3 \omega ; \omega, \omega, \omega) \mathbf{E}_{\omega} 3
\end{aligned}
$$

In silicon, the latter-representing third harmonic generation (3HG) - is of less importance for typical telecom wavelengths, as the frequency $3 \omega$ will be absorbed. In principle, this effect could lead to additional absorption although this was not yet reported so far. The former describes two effects which influence the input signal itself: the Kerr effect and degenerate two-photon absorption (TPA) related to its imaginary part.

The optical Kerr effect is related to the real part of $\chi^{(3)}$. It represents a quadratic field dependence of the refractive index, given by

$$
n=n_{0}+n_{2}|E|^{2}
$$

with $n_{0}$ the linear refractive index and $n_{2} \equiv 3 /\left(8 n_{0}\right)$. $\operatorname{Re} \chi^{(3)}(-\omega ; \omega,-\omega, \omega)$ the Kerr coefficient. Equivalently, this corresponds to the linear intensity dependence

$$
n=n_{0}+n_{2, I} I
$$

with $I$ the intensity. Using the relation $I=1 / 2 \cdot \epsilon_{0} c n_{0}|E|^{2}$, one has $n_{2, I}=2 /\left(\epsilon_{0} c n_{0}\right) \cdot n_{2}$. Potential applications of this effect include self-phase modulation leading to all-optical switching and soliton propagation.

The degenerate TPA is related to the imaginary part of $\chi^{(3)}$, representing a quadratic field or linear intensity dependence of the total absorption:

$$
\alpha_{\mathrm{tot}}=\alpha+\beta|E|^{2}=\alpha+\beta_{I} I
$$

with $\alpha$ the linear absorption coefficient and $\beta=-3 \omega /$ $\left(4 n_{0} c\right) \cdot \operatorname{Im} \chi^{(3)}(-\omega ; \omega,-\omega, \omega)$ the two-photon absorption coefficient. Again, $\beta_{\mathrm{I}}=2 /\left(\epsilon_{0} c n_{0}\right) \cdot \beta$. TPA can also be used for all-optical switching purposes.

In the presence of two input signals with frequencies $\omega_{1}$ and $\omega_{2}$, the number of third-order nonlinear polarizations grows vastly. The most important contributions are:

$$
\begin{aligned}
\mathbf{P}_{\omega_{2}} & =\epsilon_{0} \frac{3}{2} \chi^{(3)}\left(-\omega_{2} ; \omega_{1},-\omega_{1}, \omega_{2}\right)\left|E_{\omega_{1}}\right|^{2} \mathbf{E}_{\omega_{2}} \\
\mathbf{P}_{2 \omega_{1}-\omega_{2}} & =\epsilon_{0} \frac{3}{4} \chi^{(3)}\left(-2 \omega_{1}+\omega_{2} ; \omega_{1}, \omega_{1},-\omega_{2}\right) \mathbf{E}_{\omega_{1}} 2 \mathbf{E}_{\omega_{2}}^{*}
\end{aligned}
$$

and vice versa. The former nonlinear polarization is representative for the non-degenerate Kerr and TPA effect, while the latter polarization term represents an example of four-wave mixing (FWM).

The non-degenerate variants of the optical Kerr and TPA effect are two times larger than in the degenerate case. Now, the refractive index and absorption coefficient are changed by a second, pump signal instead of by the signal itself, leading to cross-phase and cross-absorption modulation.

In the FWM case, the real part of the nonlinear susceptibility gives rise to a transfer of energy from the two pump beams to a new frequency through a sort of phase grating, while the imaginary part leads to nonlinear loss. In contrast to the other nonlinear mechanisms mentioned so far, FWM processes are not automatically phasematched leading to the additional requirement: $k_{2 \omega_{1}-\omega_{2}}=2 k_{\omega_{1}}-k_{\omega_{2}}$. Possible applications include wavelength conversion and phase conjugation.

For frequencies $\omega_{1} \approx \omega_{2}$, the values of $\chi^{(3)}\left(-\omega_{2} ; \omega_{1}\right.$, $\left.-\omega_{1}, \omega_{2}\right)$ and $\chi^{(3)}\left(-2 \omega_{1}+\omega_{2} ; \omega_{1}, \omega_{1},-\omega_{2}\right)$ are in good approximation $^{17)}$ equal to that of $\chi^{(3)}(-\omega ; \omega,-\omega, \omega)$ so that measuring $n_{2}$ and $\beta$ suffices to determine the nonlinear coefficients of the different processes.

In the presence of three or more input signals, even more combinations are possible, of which the most interesting ones are extensions of the FWM example above.

\subsection{Measured values}

Different groups have measured the nonlinear coefficients of silicon around the telecom wavelength $1.55 \mu \mathrm{m}$. The obtained values are summarized in Table II. Dinu et al. ${ }^{18)}$ also reported the presence of nonlinear anisotropy.

To determine the relative strength of the real and imaginary parts of the nonlinear susceptibility, a figure of merit was defined as $F O M=n_{2} /(\beta \lambda):^{19)}$ e.g., a nonlinear phase shift of $\pi$ can be obtained with an acceptable signal degradation due to TPA for $F O M>1 / 2$. For the measurement results of Table II, a large variation of figure of merit values is obtained, leaving it still undetermined whether silicon meets the condition $F O M>1 / 2$ or not.

\subsection{Secondary effects}

The presence of nonlinear absorption effects in silicon at $1.55 \mu \mathrm{m}$ leads to the creation of free carriers, which is unexpected in the linear regime. Especially for large input signals, this free-carrier density can grow rapidly, leading to additional nonlinear effects such as free-carrier dispersion (FCD) and free-carrier absorption (FCA). With $N$ and $P$

Table II. Measured nonlinear coefficients.

\begin{tabular}{lcccc}
\hline Affiliation & $\begin{array}{c}\lambda \\
(\mu \mathrm{m})\end{array}$ & $\begin{array}{c}n_{2, \mathrm{I}} \\
\left(\mathrm{cm}^{2} / \mathrm{W}\right) \\
\times 10^{-13}\end{array}$ & $\begin{array}{c}\beta_{\mathrm{I}} \\
(\mathrm{cm} / \mathrm{W}) \\
\times 10^{-9}\end{array}$ & FOM \\
\hline $\mathrm{NEC}^{59)}$ & 1.5 & 1.45 & 0.60 & 1.61 \\
$\mathrm{CUHK}^{22)}$ & 1.54 & 0.60 & 0.45 & 0.87 \\
$\mathrm{UBC}^{23)}$ & 1.53 & 0.70 & 0.90 & 0.51 \\
Bell $^{18)}$ & 1.54 & $0.43-0.45$ & $0.79-0.88$ & $0.32-0.37$ \\
$\mathrm{UCLA}^{(6)}$ & 1.54 & - & 0.44 & - \\
$\mathrm{NTT}^{58)}$ & 1.55 & 0.90 & - & - \\
\hline
\end{tabular}


respectively the free electron and hole density, one has in silicon:

$$
\begin{aligned}
& \Delta \alpha^{\mathrm{FCA}}(N)=\sigma_{a, N} N+\sigma_{a, P} P \\
& \Delta n^{\mathrm{FCD}}(N)=-\sigma_{r, N} N-\left(\sigma_{r, P} P\right)^{0.8}
\end{aligned}
$$

with $\sigma_{a, N}=8.5 \times 10^{-18} \mathrm{~cm}^{2}, \sigma_{a, P}=6.0 \times 10^{-18} \mathrm{~cm}^{2}, \sigma_{r, N}=$ $8.8 \times 10^{-22} \mathrm{~cm}^{3}$ and $\sigma_{r, P}=4.6 \times 10^{-22} \mathrm{~cm}^{3}$ at $1.55 \mu \mathrm{m}$.

In nanophotonic structures, the lifetime of these additional carriers is largely determined by surface recombination (process dependent). Values of 1-10 ns were reported so far $^{1,20)}$ in photonic wires. However, different techniques exist which may further reduce this recombination lifetime.

One technique is carrier recombination induced by deep recombination centers. By means of proton bombardment, deep recombination centers can be produced, which effectively recombine the carriers although additional loss may be introduced due to damage to the structure.

Another possibility is carrier sweeping by applying an external dc electric field. Recently, this technique was used to create fast $\mathrm{Si}$ modulators with recovery times down to 50100 ps. $^{20,21)}$

When these carriers are not extracted, their recombination may lead to additional heating of the nonlinear device and thus resulting in a refractive index change, given by:

$$
\Delta n^{\text {heat }}(T)=\left.\frac{d n}{d T}\right|_{T=T_{0}}\left(T-T_{0}\right)
$$

with $T$ the temperature and $T_{0}$ the ambient temperature. For photonic wire-based structures, thermal relaxation times of the order of $100 \mathrm{~ns}$ have been reported. ${ }^{20)}$

Clearly, these secondary effects can complicate and degrade potential ultrafast nonlinear operation. On the other hand, they can be used themselves for all-optical signal processing with typically lower optical powers, but also with much lower bit rates.

\section{Fabrication}

To define patterns, electron beam lithography (EBL) and deep UV (DUV) optical lithography are currently used. The resist patterns are then transferred to a hard mask and the silicon or directly to the silicon by a variety of etch processes. Finally, a top cladding (oxide) can be applied.

Table III gives an overview of the methods used by several groups. Most research groups use EBL, because of the high resolution, relatively rapid prototyping and availability of EBL machines at many sites. DUV lithography, available in CMOS lines and important from a real-world application point of view as it is a mask-based process, is used at IMEC, ${ }^{5)}$ LETI, $^{24)}$ and by MIT. ${ }^{25)}$ While the resolution offered by DUV lithography is not as good as that of EBL, it can write a very large field within just one fast lithography step. However, in research environments, the total turnaround time between circuit design and obtaining fabricated structures is shorter with EBL.

In the etching step, most groups use an oxide or metal hard mask. The silicon is then etched using a dry etch process; most groups use an ICP or ECR-based processes as these can deliver smooth sidewalls with little damage. After etching, a (thermal) oxidation in gas phase ${ }^{26}$ ) or wet oxidation $^{25)}$ step can be applied to reduce the sidewall

\begin{tabular}{|c|c|c|c|}
\hline Affiliation & Litho & Mask & Etch \\
\hline $\mathrm{IMEC}^{5)}$ & $\begin{array}{c}\text { DUV } \\
248 \mathrm{~nm}\end{array}$ & Resist & $\begin{array}{c}\text { ICP-RIE } \\
\left(\mathrm{Cl}_{2} / \mathrm{He} / \mathrm{HBr} / \mathrm{O}_{2}\right)\end{array}$ \\
\hline $\mathrm{IBM}^{6)}$ & EB & Oxide & $\begin{array}{c}\text { Mask: } \mathrm{CF}_{4} / \mathrm{CHF}_{3} / \mathrm{Ar} \\
\mathrm{Si}: \mathrm{HBR}\end{array}$ \\
\hline Cornel1 $^{27)}$ & EB & $?$ & ICP-RIE \\
\hline $\mathrm{NTT}^{10,58)}$ & $\mathrm{EB}$ & $\mathrm{SiO}_{2}$ & $\begin{array}{c}\text { Mask: } \mathrm{RIE}\left(\mathrm{SF}_{6} / \mathrm{CF}_{4}\right) \\
\text { Si: ECR }\end{array}$ \\
\hline Yokohama $^{13,35)}$ & EB & Metal & ICP-RIE $\left(\mathrm{XF}_{4}+\mathrm{Xe}\right)$ \\
\hline $\operatorname{MIT}^{25)}$ & $\begin{array}{c}\text { DUV } \\
248 \mathrm{~nm}\end{array}$ & Resist & $\begin{array}{c}\text { ICP-RIE } \\
\left(\mathrm{Cl}_{2} / \mathrm{He} / \mathrm{HBr} / \mathrm{O}_{2}\right) \\
+ \text { wet oxidation }\end{array}$ \\
\hline $\mathrm{LETI}^{24)}$ & $\begin{array}{c}\text { DUV } \\
193 \mathrm{~nm}\end{array}$ & Oxide & ICP-RIE (HBr) \\
\hline Columbia $^{14)}$ & EB & $\mathrm{Al}$ & $\mathrm{RIE}\left(\mathrm{CF}_{4} / \mathrm{Ar}\right)$ \\
\hline $\mathrm{NEC}^{34)}$ & EB & $?$ & ICP-RIE \\
\hline Aachen $^{53)}$ & EB & resist & ICP-RIE (HBr) \\
\hline
\end{tabular}
roughness. Many different oxidation processes are possible.
Table III. Fabrication methods.

Table IV. Inverse taper fiber couplers.

\begin{tabular}{lcccr}
\hline Affiliation & Fabrication & Cladding & Fiber & Loss \\
\hline $\mathrm{IBM}^{6)}$ & EBL & Polymer & high NA & $<0.5 \mathrm{~dB}$ \\
\hline $\mathrm{NTT}^{10,58)}$ & EBL & $\begin{array}{c}\text { Polymer } \\
\text { SiON+oxide }\end{array}$ & $\begin{array}{c}\text { high NA } \\
\text { high NA }\end{array}$ & $0.8 \mathrm{~dB}$ \\
& & $\mathrm{SiO}_{2}$ & high NA & $<4 \mathrm{~dB}$ \\
\hline Cornell $^{27)}$ & EBL & $\begin{array}{c}\mathrm{BCB}+ \\
\text { polyimide }\end{array}$ & lensed & $1.9 \mathrm{~dB}$ \\
\hline $\mathrm{IMEC}^{28)}$ & $\mathrm{DUV}$ & & \\
\hline
\end{tabular}

At IMEC, deep UV lithography with a $248 \mathrm{~nm}$ illumination wavelength is currently used, with resist as the etch mask for an ICP etch. The process steps are described in detail in ref. 5. Optionally, we apply a top cladding of $\mathrm{SiO}_{2}$ or another material.

\subsection{Coupling to fiber and pigtailing}

Coupling from submicron wire waveguides to singlemode fibre was long seen as one of the major problems. However, today, sub-dB broadband coupling losses with "inverted tapers" have been demonstrated by multiple groups. Table IV gives an overview. In this scheme, the wire is narrowed to a very small tip of just a few tens of nm wide, pushing the mode out of the core into a low-index overlay. At the end of the taper, both the mode size and the effective index are much better matched to the fiber mode. Still, in most cases, high NA single-mode fiber with a reduced mode size compared to standard fiber needs to be used to obtain low coupling losses. As the low index overlay, polymer ${ }^{6,10)}$ or inorganic materials ${ }^{10,27)}$ can be used. The latter may be more reliable and more suited for nonlinear applications needing high input powers, while a polymer overcladding is potentially cheaper and easier to deposit. Most groups ${ }^{6,10,27)}$ pattern the very small taper tip with EBL. Roelkens et al. ${ }^{28)}$ have adapted the scheme in order to support the more limited resolution of $248 \mathrm{~nm}$ DUV lithography, and obtained a fiber-to-waveguide coupling losses of $1.9 \mathrm{~dB}$.

A different scheme is the vertical fiber coupler. Here, a 
high index contrast grating is used to scatter the incident waveguide mode to the fiber. Simple uniform gratings in our SOI stack show a fiber-to-waveguide loss of $4.5 \mathrm{~dB}$ when index-matching material is applied between fiber and coupler. In order to obtain a higher coupling efficiency, one must use a more complex grating and eliminate the refraction order that is directed into the substrate, for instance by applying a bottom mirror. ${ }^{29)}$ The main advantages of this coupling method are the large alignment tolerances, the possibility of wafer-level testing, and the coupling to standard single mode fiber. We demonstrated a pigtailed SOI device with an eight-fiber array coupler based on this principle. ${ }^{30)}$ Using a 2D extension of the vertical fiber coupler scheme, one obtains a polarization-splitting fiber coupler, where both fiber polarizations are coupled to separate waveguides. From the point of view of the waveguides, both polarizations are TE. ${ }^{31)}$ In this way one can resolve the polarization dependency problem of silicon wire waveguide circuits.

\section{Linear Circuits and Circuit Elements}

With the current state of technology, circuit length and performance are limited by losses and crosstalk. As discussed in $\S 2$, dispersion will not limit the interconnect length within an SOI wire based photonic integrated circuit (PIC) and the interconnects as such can support several Tbit/s per wavelength. However, some circuit elements such as ring resonators may have a very high dispersion at the operation wavelength so that dispersion can become important when designing a circuit.

Losses arise mostly from excess losses in components such as splitters, intersections, couplers, and multimode interferometers (MMI's). Currently, these excess losses are of the order of a few tenths of $d B$. While this is not a problem for a simple component, it very quickly becomes a bottleneck in larger circuits. This is intrinsic to the high index contrast as such and of the state of fabrication technology. First of all, one needs careful optimization of the design, which is harder at high index contrasts. Secondly, high index contrast also means inevitable reflections and scattering to radiative modes even with very careful optimization, e.g., at interfaces between waveguides of different width such as in an MMI. This may require to either locally reduce the width differences or locally reduce the index contrast. A third problem is the current state of technology, especially with the more limited resolution of DUV lithography but even with EBL, limiting the possible structure detail and leading to fabrication variations.

For communication applications, crosstalk is a second problem. Again, the high index contrast makes the structures harder to optimize and makes them having an intrinsically higher crosstalk than lower index contrast structures. Also, fabrication variations and limited resolution have a large impact. This not only holds for wavelength-selective elements, but also for intersections which can lead to very high crosstalk.

In the following sections we will briefly review and discuss some basic components.

\subsection{Intersections}

Intersections between waveguides are inevitable in many planar circuits even with a limited number of inputs and outputs. An example is a cross-connect consisting of an AWG and switches, where even with only two inputs and two outputs, waveguide crossings cannot be avoided. Simple intersections were calculated to have a $1.4 \mathrm{~dB}$ loss and a crosstalk of up to $-9 \mathrm{~dB}^{32)}$ Generally, the total number of intersections in one input to output path can be limited. This makes the loss acceptable, but still the crosstalk generated can be too high for communication applications. The elimination of crosstalk in waveguide intersections has been studied by Johnson et al. ${ }^{33)}$ By altering a weakly resonant cavity at the cross-section, one can obtain high transmission and low crosstalk. Of course, the bandwidth of the crosssection depends on the quality factor of the cavity and will degrade with better transmission.

By tapering the waveguides, the beam incident on the intersection becomes less divergent and one can expect the crosstalk due to direct coupling to become lower. Also, the impedance mismatch between the waveguide and the intersection is smaller, giving less reflections and scattering to radiative modes. The resonance at the intersection is kept weak, giving a large bandwidth. Measurement results with EBL fabricated structures have shown $1.2 \mathrm{~dB}$ loss, ${ }^{32)}$ and a reduced crosstalk of $-25 \mathrm{~dB}$ for an asymmetrical intersection. In DUV fabricated structures, we showed $0.65 \mathrm{~dB}$ loss and $-30 \mathrm{~dB}$ crosstalk by measuring series of multiple intersections. ${ }^{8)}$ The tapers can be just $5 \mu \mathrm{m}$ long, therefore the impact on circuit size is limited. While these results were close to the simulated values, it is clear that still only a few intersections can be supported so losses need to be reduced further in order to avoid an intersection bottleneck. Locally using a lower index contrast may help avoiding the problem.

\subsection{Couplers and splitters}

In couplers and splitters, fabrication errors can make the splitting or coupling ratio deviate from its designed value, leading to crosstalk and excess loss in wavelength-selective elements and imbalance in power division circuits.

Directional couplers in wires with square cross-section were studied in detail by Yamada et al. ${ }^{34)}$ The coupling length was just 5 to $10 \mu \mathrm{m}$ for 200 to $300 \mathrm{~nm}$ waveguide spacing. The extinction ratio achieved was larger than $20 \mathrm{~dB}$.

Sakai et al. ${ }^{35)}$ studied several designs for compact $3 \mathrm{~dB}$ couplers theoretically and experimentally. A simple branch consisting of two offset bends turned out to have the lowest insertion loss while staying manufacturable. Simulations predicted $0.2 \mathrm{~dB}$ excess loss for such a structure, while experimentally $0.3 \mathrm{~dB}$ was measured. The splitter was used in a three level $\mathrm{H}$-tree, with a measured fluctuation of $2-$ $5 \mathrm{~dB}$ over the 16 output ports. $\left.{ }^{36}\right)$

MMI-type $3 \mathrm{~dB}$ couplers were experimentally demonstrated by Orobtchouk et $a .^{24)}$ and a 1-to-8 (three level) distribution tree was demonstrated. The imbalance remained smaller than $0.5 \mathrm{~dB}$ over a $400 \mathrm{~nm}$ spectral range. The size of the $1 \times 2$ MMI was only $2 \times 5.4 \mu \mathrm{m}^{2}$.

We fabricated Y-type and MMI-type $3 \mathrm{~dB}$ splitters with DUV lithography. ${ }^{8}$ ) From the extinction ratio of MachZehnder interferometers, we estimated the imbalance to be smaller than $0.13 \mathrm{~dB}$ for the $\mathrm{Y}$ splitters. These still had an excess loss of around $1.5 \mathrm{~dB}$ however. A $3 \times 7.6 \mu \mathrm{m}^{2} \mathrm{MMI}$ shows an insertion loss smaller than $1 \mathrm{~dB}(0.5 \mathrm{~dB} /$ port $)$ and 
very good balance. Splitter trees with up to $1 / 64$ splitting ratio (six level) were fabricated and show a very good balance.

\section{Linear Wavelength Filter Devices}

For communication purposes, SOI wires offer an attractive way to implement filter devices such as channel drop filters, multiplexers and demultiplexers. The implementation of devices through different approaches has been reported, such as Bragg grating based-devices consisting of gaps etched in wires or fin-like structures mounted on wires, showing promising experimental results. ${ }^{37,38)}$ Foresi et al. investigated photonic bandgap structures with a microcavity defect embedded in wires. ${ }^{39)}$ Kazmierczak et al. demonstrated an add-drop filter type consisting of two microdisc resonators mounted on two crossing wires. ${ }^{40)}$ In the following, we will focus on Mach-Zehnder interferometers, lattice filters, arrayed waveguide gratings, and ring resonators devices performing such tasks.

\subsection{Mach-Zehnder interferometer}

The Mach-Zehnder interferometer (MZI) is generally used as a broadband channel selector filter, or as a building block for more complex filters, or when electrically contacted, for switches and modulators. There are however amazingly few reports on this popular device in SOI wires. Ohno et al. ${ }^{41)}$ fabricated $1 \times 1,1 \times 2$, and $2 \times 2$ MZIs. The $1 \times 1$ MZI had a $20.5 \mathrm{~dB}$ extinction ratio, showing that the splitting ratio of the $\mathrm{Y}$-splitters used was very well balanced. The $1 \times 2$ and $2 \times 2$ MZI devices had maximum extinction ratios of the same order. However, the bend-waveguide couplers had splitting ratios deviating from 0.5 , leading to a $5 \mathrm{~dB}$ difference in extinction ratios for both output ports. Still, it was the first report of MZI devices in SOI wires.

We fabricated $1 \times 1$ MZI devices ${ }^{8)}$ using Y-splitters and delay lengths from $20 \mu \mathrm{m}$ up to $1 \mathrm{~mm}$ in one arm, corresponding to a $27 \mathrm{~nm}$ down to $550 \mathrm{pm}$ free spectral range. The splitters still had quite some excess loss, partly due to unoptimized design. The splitter unbalance was estimated to be smaller than $0.13 \mathrm{~dB}$. Figure 4 shows the measured transmission of a MZI with a $1 \mathrm{~mm}$ delay length in one arm. The extinction ratio reaches $30 \mathrm{~dB}$.

\subsection{Mach-Zehnder lattice filter}

Mach-Zehnder lattice filters can possibly yield very compact channel drop filters. At WDM channel spacings, their size is determined by the delay length needed and by the coupler lengths. Lattice filters intrinsically have large channel bandwidths, but need several stages in order to achieve a large enough extinction ratio. To design the coupling ratios, a number of approaches are possible. A good extinction ratio at a specified channel bandwidth requirement is obtained by a digital filter design based method minimizing the error between the designed and desired filter characteristic in the Chebyshev sense. ${ }^{42)}$ Deviations from the designed couplers due to fabrication errors can however introduce significant crosstalk.

We demonstrated lattice channel drop filters with a discrete set of different coupling coefficients. ${ }^{43}$ A device with a $17 \mathrm{~nm}$ FSR, $2.6 \mathrm{~nm}$ channel bandwidth and a very low insertion loss was demonstrated. The extinction ratio in the

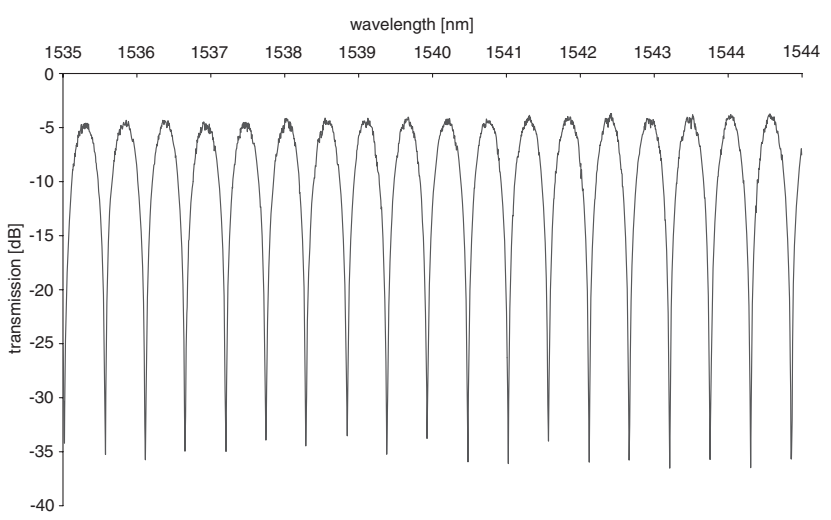

Fig. 4. Normalized transmission of a $1 \times 1$ MZI with a $1 \mathrm{~mm}$ delay length in one arm.

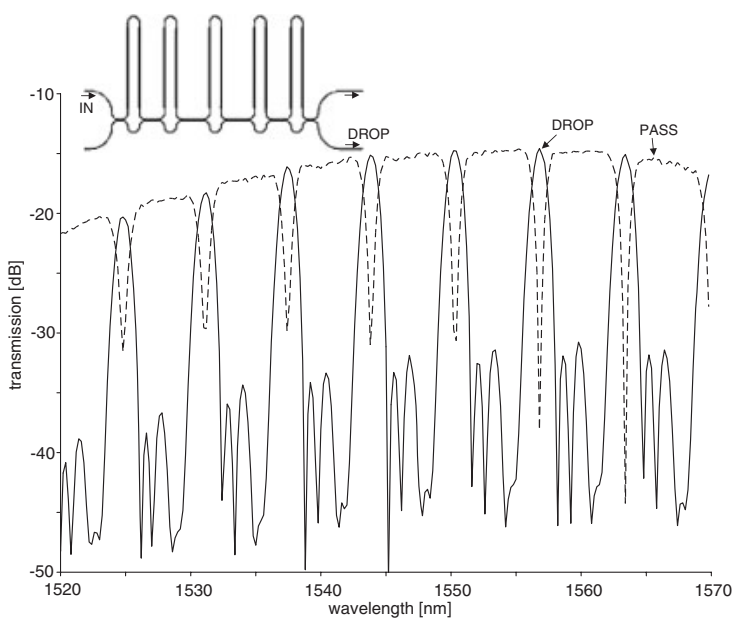

Fig. 5. Fiber-to-fiber transmission spectra of dropped and passed signal in a lattice filter with $200 \mathrm{GHz}$ channel spacing. The slow parabolic variation of the loss is due to the vertical fiber couplers.

throughput channel was 13 to $20 \mathrm{~dB}$, but the sidelobes in the dropped spectrum were up to $-10 \mathrm{~dB}$, giving a quite high crosstalk. More recently, we fabricated several devices with a Chebyshev minimization design and WDM compatible channel spacings. Figure 5 shows the measured spectrum of a 1-from-4 channel selector with a $200 \mathrm{GHz}$ channel spacing. The device has six directional couplers. The extinction ratio for the dropped channel is again 13 to more than $20 \mathrm{~dB}$, and the sidelobe level has been reduced to $-16.5 \mathrm{~dB}$. In order to achieve this, the straight delay line waveguides were broadened to $800 \mathrm{~nm}$, reducing the phase errors due to small width variations. The residual sidelobe level is due to systematic and random coupler deviations and residual phase errors.

Tsuchizawa et $a l .{ }^{10)}$ have demonstrated lattice filters using bend radii of $2.5 \mu \mathrm{m}$. These had a very large FSR of $84 \mathrm{~nm}$, a $10 \mathrm{~nm}$ channel bandwidth and a drop insertion loss of $2 \mathrm{~dB}$. The crosstalk was still $-12 \mathrm{~dB}$ however. By apodizing the couplers, the authors improved the device to a $-19 \mathrm{~dB}$ crosstalk level. ${ }^{44)}$

\subsection{Arrayed waveguide grating}

Arrayed waveguide grating (AWG) devices are popular filter elements because of their versatility and the many 
channels that can simultaneously be operated on. By reducing the bend radius to just a few $\mu \mathrm{m}$, the size of an AWG can considerably be reduced. However, losses in the star couplers and crosstalk due to unoptimized design and fabrication variations are a problem with SOI wires. We are studying AWG devices with WDM-compatible channel spacings. Earlier, we demonstrated a first AWG router, still with a classical layout, device insertion losses of $8 \mathrm{~dB}$ and a high crosstalk of $-7 \mathrm{~dB} .{ }^{45)}$ Because most of the losses are due to scattering at the star couplers, we reduced the index contrast of the star couplers by applying a two-step etching process. ${ }^{30)}$ This resulted in a reduced insertion loss of $3.5 \mathrm{~dB}$. However, the crosstalk was still $-12 \mathrm{~dB}$ for the best sample. We think most of the crosstalk is due to nm-scale width variations and roughness in the arrayed waveguides. When broadening these waveguides so that the propagation constant does not change as much with waveguide width, we see a reduced crosstalk level, as with the lattice filters. This resulted in an 16-channel AWG with a $200 \mathrm{GHz}$ channel spacing, $2.2 \mathrm{~dB}$ insertion loss, -15 to $-20 \mathrm{~dB}$ sidelobes and a footprint of just $0.1 \mathrm{~mm}^{2}$. This device has 36 arrayed waveguides. The overlayed transmission spectra of a central input to the 16 outputs is shown in Fig. 6.

Fukazawa et al. ${ }^{46)}$ demonstrated a very compact AWG demultiplexer of only $110 \times 93 \mu^{2}$ footprint with a $6 \mathrm{~nm}$ channel spacing and a free spectral range larger than $90 \mathrm{~nm}$. Half-elliptical tapers were used between the waveguides and slab in order to reduce the insertion losses. The sidelobe level still was $-5 \mathrm{~dB}$. Note that reducing the channel spacing requires a larger device. From the same group, Sasaki et $a l .{ }^{47)}$ reported on an AWG with an even smaller footprint but similar large free spectral range. The device was optimized using FDTD. This resulted in a very low reported insertion loss $(<1 \mathrm{~dB})$, however normalized to the average propagation loss in a grating arm. The best crosstalk value was $-13 \mathrm{~dB}$.

\subsection{Ring resonator}

Ring resonators are a basic component for many interesting optical functions. Coupled to two waveguides, they can serve as add-drop filters. Due to the resonant nature, multiple coupled rings need to be used in order to obtain a high enough passband width for communication applications. However, single ring resonators could serve as very sensitive integrated sensors. Their highly dispersive nature can be used in more complex wavelength filters, or devices such as dispersion compensators.

The great advantage of SOI ring resonators is the very large free spectral range that can be achieved with a single ring. In this way, one does not need to resort to multiple rings exploiting the Vernier effect or cascaded operation. SOI ring resonators were studied by a number of groups, and high quality factor resonances have been demonstrated. Were first experiments still only achieved Q factors of 250 in a $5 \mu \mathrm{m}$ radius ring, ${ }^{48)}$ processing technology has been optimized and nowadays Q factors of several 1000 to 10000 are achieved.

Using deep UV lithography, we fabricated ring resonators with $\mathrm{Q}$ factors with high drop efficiency and throughput extinction. Racetrack resonators with $5 \mu \mathrm{m}$ radius had a throughput extinction ratio better than $20 \mathrm{~dB}$ and a 50 to

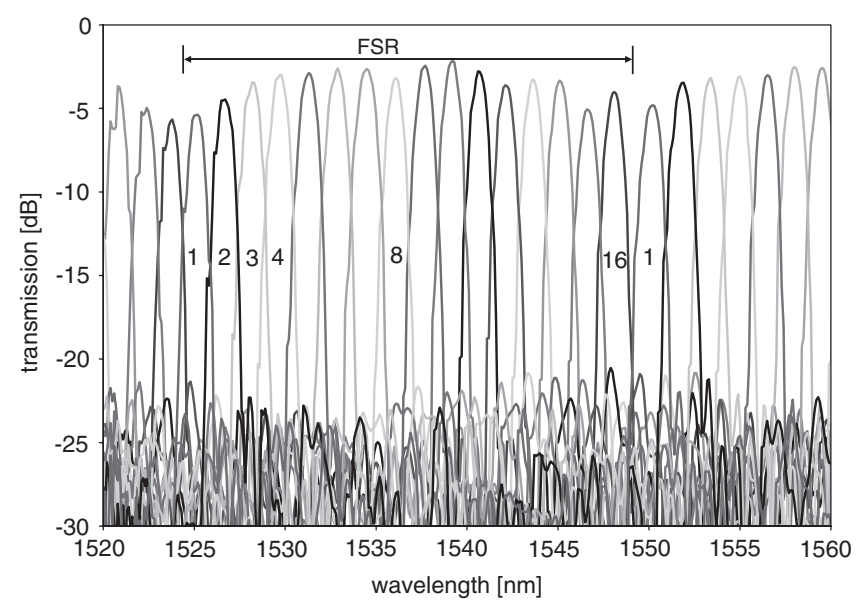

Fig. 6. Overlayed transmission spectra of a 16-channel AWG with $200 \mathrm{GHz}$ channel spacing. The transmission is normalized to give the transmission of the actual AWG.

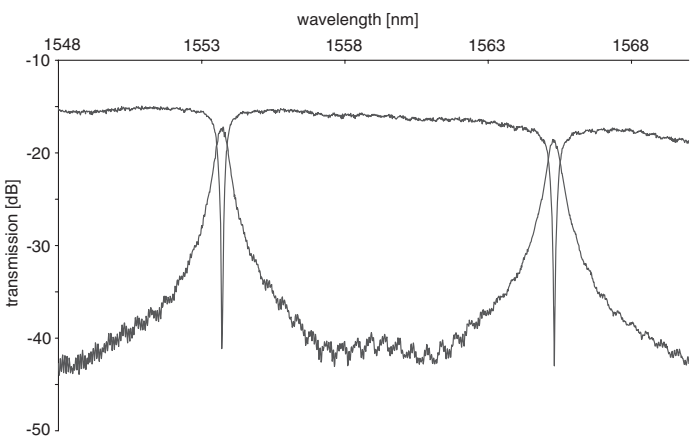

(a)

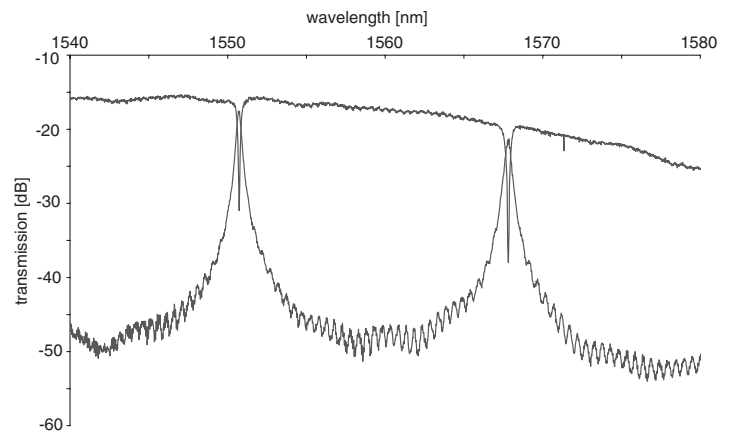

(b)

Fig. 7. Fiber-to-fiber transmission spectra of ring resonators with $5 \mu \mathrm{m}$ radius.

$70 \%$ channel drop efficiency. ${ }^{49)}$ A measurement is shown in Fig. 7. The Q factor was still larger than 3000 for a $11.5 \mathrm{~nm}$ FSR. Circular ring resonators coupled to bent waveguides can be used in order to achieve a high enough coupling while keeping the FSR large. ${ }^{50)}$ We used this technique and showed add-drop filters with a throughput extinction ratio of -10 to $-15 \mathrm{~dB}$ and high drop efficiency, with a FSR of $17 \mathrm{~nm}^{51)}$ (Fig. 7).

Tsuchizawa et al. demonstrated channel drop filters with dropped resonances having Q factors of 10000 and 20000 in 5 and $10 \mu \mathrm{m}$ radius rings, respectively. ${ }^{10)}$

Often, the extinction in the throughput signal at resonance is a problem and leads to crosstalk when the structure is used 


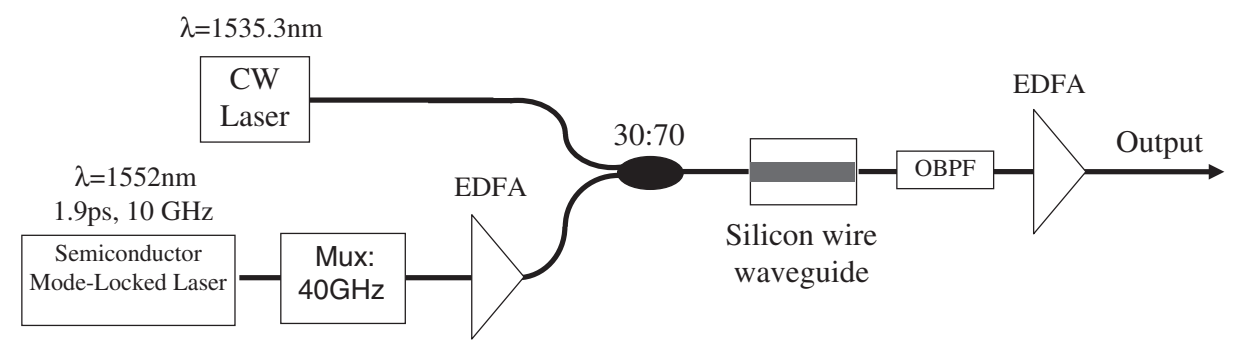

Fig. 8. Ultrafast switching in silicon wire waveguides: simplified experimental setup.

as a channel add-drop filter. Vörckel et $a .^{52)}$ studied asymmetrical coupling of a racetrack resonator to two waveguides, for improving the extinction ratio between dropped and throughput signals in an add-drop filter, at the expense of $\mathrm{Q}$ factor. The authors demonstrated an $8.8 \mathrm{~dB}$ crosstalk reduction by using asymmetrical coupling. By the same group, ring resonators with $20 \mu \mathrm{m}$ radius were demonstrated achieving very high $\mathrm{Q}$ factors up to $139000 .{ }^{53)}$ These resonators could be used as very sensitive sensors. From the transmission measurements, waveguide propagation losses of $1.9 \mathrm{~dB} / \mathrm{cm}$ were measured for $\mathrm{TM}$ polarisation.

Baehr-Jones et al. ${ }^{54)}$ demonstrated ring resonators coupled to a single waveguide, with thin SOI wires with a less confined field. These resonators with $30 \mu \mathrm{m}$ radius showed loaded Q values of 45000 and 57000. The authors also studied the tuning sensitivity of these resonators due to temperature and cladding changes. ${ }^{12)}$

\section{Nonlinear Applications}

\subsection{Nonlinear applications in wires}

In recent years, several reported studies have described excellent progress on the use of optical non-linearities in silicon wire waveguides. Significant Raman on-off gain of $0.7 \mathrm{~dB}$ from $4.2 \mathrm{~mm}$ long wire waveguides using CW pump power of $1 \mathrm{~mW}$ was obtained by Espinola et al. ${ }^{55)} \mathrm{Xu}$ et al. showed $3.1 \mathrm{~dB}$ of net Raman gain from a picosencond order pump signal with $2.8 \mathrm{~W}$ of peak power in a $7 \mathrm{~mm}$ long waveguide. ${ }^{56)}$ A wavelength conversion scheme via four wave mixing (FWM) process with conversion efficiency of approximately $-40 \mathrm{~dB}$, and tuning range from 21.6 to 108 $\mathrm{GHz}$ was reported by Espinola et al. ${ }^{57)}$ Fukuda et al. demonstrated wavelength conversion with efficiency of $-35 \mathrm{~dB}$ in a $15.8 \mathrm{~mm}$ long waveguide by FWM. ${ }^{58)}$ All optical switching with the transmission signal modulated by up to $94 \%$ in less than 500 ps using light pulses with energies as low as $25 \mathrm{pJ}$ was shown by Almeida et al. ${ }^{1)}$

In this section we give some insights into our own results obtained from TPA processes. We demonstrated several applications, such as all optical switching, ${ }^{60)}$ all optical switching in high repetition ratio regime, ${ }^{61)}$ multi-color operation of our proposed optical switching scheme, optical logic circuits, ${ }^{62)}$ and optical pulse shaping. ${ }^{63)}$

\subsection{Low energy ultrafast switching in wires}

Up to now, most of the reported silicon-based switching devices rely on the plasma dispersion effect for their operation principle. To produce the required absorption or phase shift in such devices, excess free carriers are introduced inside the waveguides by either external current injection, ${ }^{64)}$ or optical excitation. ${ }^{68)}$ Thus the obtained speed, in the order of hundreds of picoseconds, is always limited by the effective carrier lifetime. The use of modulation based on TPA induced free-carrier absorption, has also been demonstrated in silicon rib structures. ${ }^{65,66)}$ However, their speed was likewise limited in response time by carrier recombination.

We previously demonstrated that the effect of slow response time due to free carrier recombination on TPA induced free-carrier absorption may be minimized by properly fitting the pulsewidth and energy of the pump pulses with the parameters of the used waveguide. ${ }^{60)}$ Based on this principle, we have shown optical switching at $40 \mathrm{GHz}$ repetition rate in wire waveguides. ${ }^{61)}$

Figure 8 illustrates the ultrafast switching in a simplified experimental setup. A semiconductor mode-locked laser generates pump pulses of $1.9 \mathrm{ps}$ FWHM pulsewidth at $10 \mathrm{GHz}$ in the wavelength of $1552 \mathrm{~nm}$. The pump pulses are multiplexed up to $40 \mathrm{GHz}$, and then amplified before being combined with the $\mathrm{CW}$ probe signal, that is at the wavelength of $1535.3 \mathrm{~nm}$. The combined signals are coupled into the silicon waveguide. An optical bandpass filter (OBPF) suppresses the pump pulses after the waveguide, allowing only the $40 \mathrm{GHz}$ modulated $\mathrm{CW}$ signal at the output.

The CW probe signal after the $\mathrm{Si}$ waveguide is inversely cross modulated by the pump pulses. After suppression of the remaining pump signal, the $\mathrm{CW}$ light appears at the output in the form of dark pulses. Due to the insufficient response of the $50 \mathrm{GHz}$ photodetector we used, the real modulation depth could not be measured by direct detection and observation on the oscilloscope. The spectrum of the $40 \mathrm{GHz}$ modulated $\mathrm{CW}$ signal is shown in Fig. 9. The clear spectral lines with $40 \mathrm{GHz}$ spaced from the $\mathrm{CW}$, the center peak, emphasize the efficiency of the modulation.

To measure the real modulation depth we performed time resolved two-color pump-probe measurements. The experiments were performed with the probe and pump signals spectrally sliced from a broadband stretched pulse passive mode-locked fiber laser, with a repetition ratio of $50 \mathrm{MHz}$. Both probe and pump signals had a pulse width of $1.5 \mathrm{ps}$. The probe signal coupled into the waveguide was $-5 \mathrm{dBm}$ in power, and the pump pulse energy was less than $3 \mathrm{pJ}$. The results of the experiments, shown in Fig. 10, indicate that $92 \%$ of modulation depth can be achieved at such low switching energy. The switching time is about $3 \mathrm{ps}$ (FWHM). From the results shown in this figure, we also confirmed the absence of the slow response due to carrier recombination. 


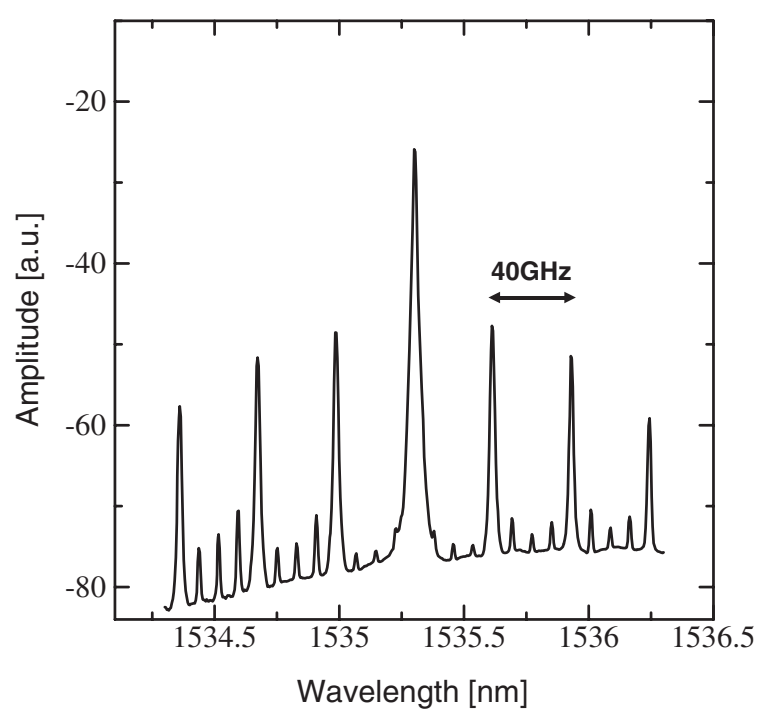

Fig. 9. Optical spectrum of $40 \mathrm{GHz}$ modulated $\mathrm{CW}$ signal.

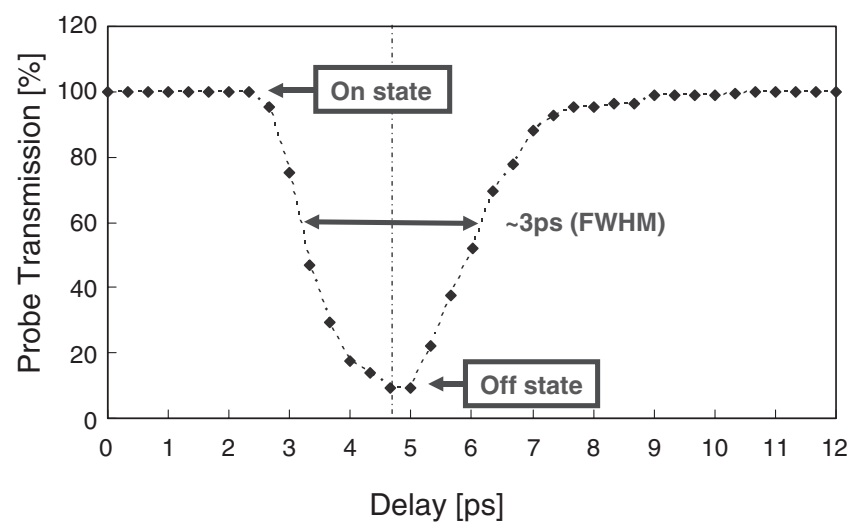

Fig. 10. Pump-probe measurements: response time.

In addition, we also experimentally demonstrated the multi-color operation of the ultra fast switching. We used a semiconductor mode locked laser with $1.7 \mathrm{ps}$ FWHM pulsewidth at $10 \mathrm{GHz}$ in the wavelength of $1535 \mathrm{~nm}$ to simultaneously modulate five $\mathrm{CW}$ probe signals, which were at the wavelengths of $1548.52,1554.13,1559.79,1565.5$, and $1571.24 \mathrm{~nm}$ respectively. Figure 11(a) shows the time domain trace of modulated CW1 in the form of dark pulses after the suppression of the pump pulses and other CW signals. The shown pulses here are broadened to about $13 \mathrm{ps}$ due to the response of the $50 \mathrm{GHz}$ photodetector used. The spectrum of the five modulated $\mathrm{CW}$ signals is shown in Fig. 11(b). The modulation disparity between channels is due to the lack of polarization control for each individual channel, as the light is launched in the waveguide via gratings, which accepts only TE modes. Compared to single probe operation, we found no deterioration in the modulation performance when five probe signals were used, as long as the individual relative power levels are kept the same.

\subsection{Potential of silicon in ultrafast photonic signal processing in wires}

By demonstrating an all-optical logic NOR gate based on TPA in submicron size silicon wire waveguides, ${ }^{62)}$ we have shown that silicon waveguides have potential applications in ultrafast photonic signal processing. The experimental setup of the silicon based NOR gate is shown in Fig. 12. Two signals, A and B, having the same peak power and pulsewidth of $1.5 \mathrm{ps}$ FWHM were spectrally sliced from a broadband stretched pulse passive mode-locked fiber laser with a repetition ratio of $50 \mathrm{MHz}$. Signal A was centered at $1545 \mathrm{~nm}$ and was multiplexed by a $25 \mathrm{ps}$ delay line, while signal B was centered at $1555 \mathrm{~nm}$ and was multiplexed by a 12.5 ps delay line. Signal A carried the digital signal "1010", while signal B carried the signal "0011", corresponding to a pseudo $80 \mathrm{Gbps}$ data rate. Both signals A and $\mathrm{B}$ were combined and coupled into the waveguide together with a $\mathrm{CW}$ probe signal at the wavelength of $1560 \mathrm{~nm}$. After passing through the $\mathrm{Si}$ wire waveguide, the $\mathrm{CW}$ probe light was cross-modulated by the sum of the signals A and B based on the non-degenerate TPA process. The bandpass filter after the waveguide allows only the modulated CW signal, which was detected by a $50 \mathrm{GHz}$ bandwidth photodiode. The Boolean NOR operation was achieved at the output in the form of dark pulses.

The measured signal A, signal B and the output are shown in Figs. 13(a)-13(c) respectively. The two signals A and $B$ operate at different wavelengths to avoid interference and thus ensure a stable output waveform. The pulsewidth of the signals after OBF1 and OBF2 were both measured to be 1.6 ps FWHM by an autocorrelator. However, when measured on the sampling oscilloscope the pulses were broadened to around $13 \mathrm{ps}$ due to the limited bandwidth of the photodetector, which also limited the measurements of the real modulation depth at the output. The peak powers of $\mathrm{A}$ and $\mathrm{B}$ were less than $5 \mathrm{~W}$ and the corresponding pulse energy was less than $8 \mathrm{pJ}$. Thus, based on our previously shown two-color pump-probe experiments, the modulation depth of the output dark pulse is expected to be more than

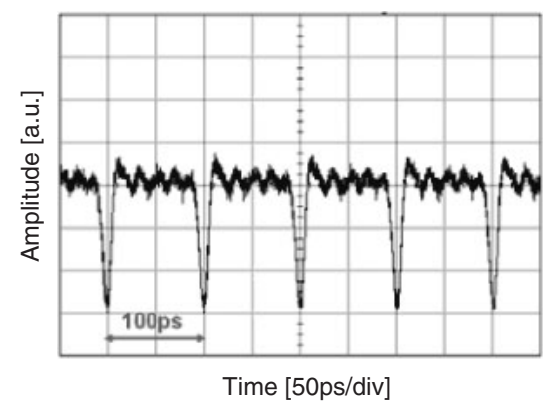

(a)

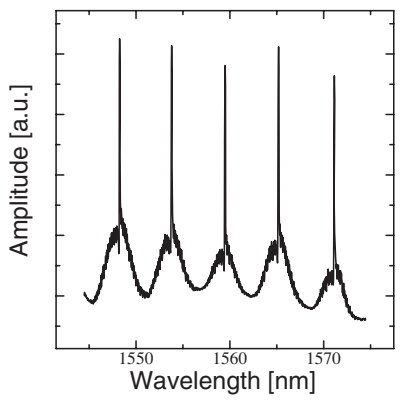

(b)
Fig. 11. (a) Time domain trace of modulated CW1 (b) Spectrum of the five modulated CW signals. 


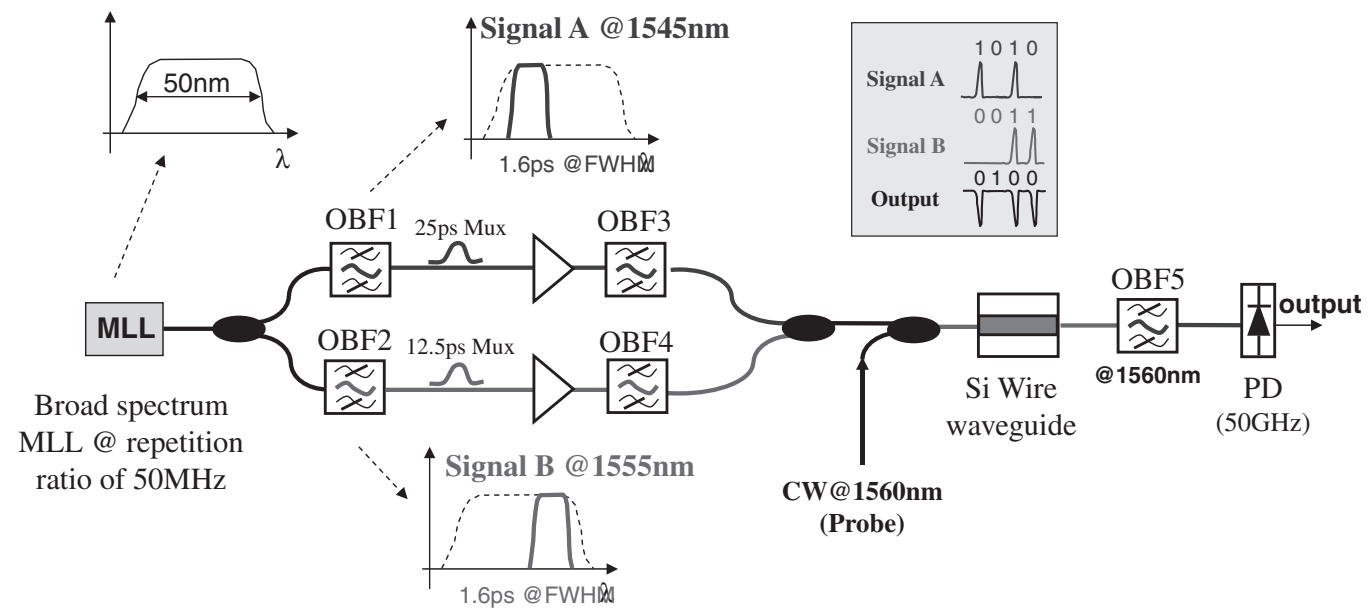

Fig. 12. Silicon based NOR gate-experimental setup.

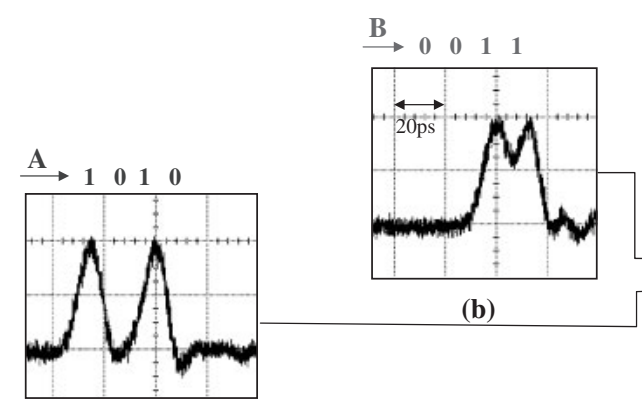

(a)

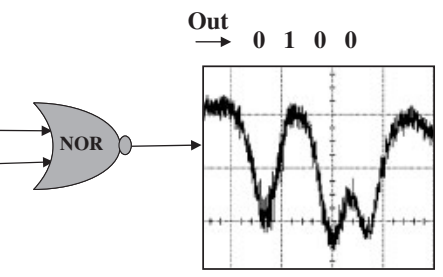

(c)
Fig. 13. (a) Signal A. (b) Signal B. (c). Output cross-modulated CW probe with logic NOR operation.
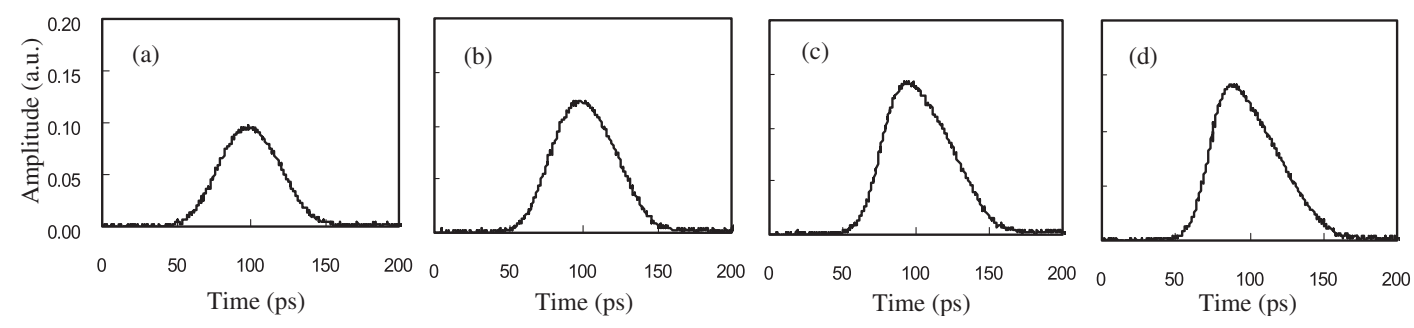

Fig. 14. Measured output pulse profiles at various peak-coupled powers. (a) $1 \mathrm{~W}$, (b) $1.6 \mathrm{~W}$, (c) $4.2 \mathrm{~W}$, and (d) $10 \mathrm{~W}$.

$90 \%$. It should be noted that the dark pulses at the output can be avoided if the $\mathrm{CW}$ probe light is replaced by a pulse train.

\subsection{Optical pulse shaping in silicon wire waveguides}

We experimentally demonstrated the temporal nonlinear transmission characteristic of high intensity picosecond pulse in silicon wire waveguides. ${ }^{63)} \mathrm{We}$ found that the generation of free carriers along the pulse duration leads to an asymmetric profile of the output pulses, which may find application in nonlinear pulse shaping devices in optical communications, such as pulse compressors or signal regenerators.

We shined pump pulses of 49 ps FWHM pulsewidth at the repetition ratio of $50 \mathrm{MHz}$ into a $2 \mathrm{~mm}$ long waveguide, whose cross section was $480 \mathrm{~nm}$ in width and $220 \mathrm{~nm}$ in height. The use of long pulsewidth allowed sufficient freecarrier generation within the pulse duration, and also enabled the pulse profile to be readily measured on a sampling oscilloscope. The maximum transmitted peak power was limited at around $1.5 \mathrm{~W}$. Further increasing the input power will not increase the peak power at the output, but will increase asymmetry in the output pulse.

Figure 14 shows the output pulse profiles at various peakcoupled powers. The asymmetry of pulse profile is clearly observed at peak powers higher than $4 \mathrm{~W}$. When the pulse was propagating along the waveguide, the leading part of the pulse generated free carriers and attenuated the trailing part of the pulse. Thus the output pulse experienced different attenuation coefficients within the pulse duration.

\subsection{Resonators}

Using optical ring resonators-which provide in an additional longitudinal confinement - the power and length requirements for ultrafast optical signal processing can even be further reduced: due to the field enhancement and the slow pulse propagation, the nonlinear interaction between 


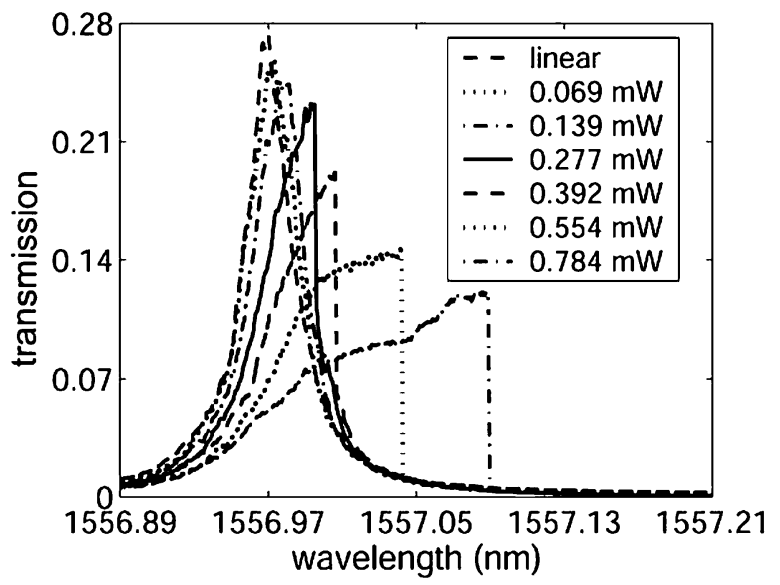

Fig. 15. Normalized transmission of the drop port for different input powers. The linear pass and drop transmissions are indicated as reference. Bistability is obtained for powers equal and above $277 \mu \mathrm{W}$.

the light and the silicon material is much larger, however typically at the cost of bandwidth. ${ }^{67)}$

So far, this was only demonstrated directly (showing the ultrafast nonresonant effect itself and not its secondary effects) for the case of FWM $\left[\chi^{(3)}\left(-2 \omega_{1}+\omega_{2} ; \omega_{1}, \omega_{1}\right.\right.$, $\left.\left.-\omega_{2}\right)\right]{ }^{58)}$ here, a conversion efficieny improvement of $8 \mathrm{~dB}$ compared to a simple wire with the same path length was obtained by using a ring resonator with a $5 \mu \mathrm{m}$ radius and a Q-factor of 10000 .

Most experiments were however performed to determine the impact and potential of secondary effects in these resonant structures: using an all-pass ring configuration with a radius of $5 \mu \mathrm{m}$ and $\mathrm{Q}$ of about 14000 , Almeida et al. ${ }^{68)}$ showed for the first time all-optical bistability with an input power of $800 \mu \mathrm{W}$. The origin was verified to be thermal dispersion $(\Delta n>0)$ and all-optical memory operation was demonstrated. In addition, all-optical modulation of $94 \%$ was obtained with an input power of $2.5 \mathrm{~W}$ (pulse length $10 \mathrm{ps})$ based on free-carrier effects $(\Delta n<0) .{ }^{1)}$

Using an add-drop ring configuration with radius of $4 \mu \mathrm{m}$ and $\mathrm{Q}$ of 51000 , we demonstrated thermal bistability with $\mathrm{CW}$ input powers below $300 \mu \mathrm{W} .^{20)}$ This is shown in Fig. 15 (only the upper arm is plotted). Analysis showed that thermal effects are indeed dominant, but a large contribution of free carriers is also present, in agreement with the results of Almeida. By measuring the standard deviation on the signal, evidence of quasi-periodical pulsations was obtained, which originates from the fact that the free-carrier and thermal dispersion have opposite signs and different time constants. ${ }^{69)}$ This is plotted in Fig. 16 for an input power of $0.76 \mathrm{~mW}$. Such a behavior may limit potential switching and memory operations, but also provides possibilities for pulse generation.

By modulating the pump signal and placing a $\mathrm{CW}$ probe signal near a second resonance (with a Q of 19000), we also demonstrated true and inverse wavelength conversion based on FCD, depending on the exact position of the probe signal. This is shown in Fig. 17 for a peak input power of $660 \mu \mathrm{W}$ and an extinction ratio of $5 \mathrm{~dB}$. A $10001000 \ldots$ data pattern $(0.1 \mathrm{~Gb} / \mathrm{s})$ was used to demonstrate the true and inverse character of the conversion: one obtains $10001000 \ldots$ on the

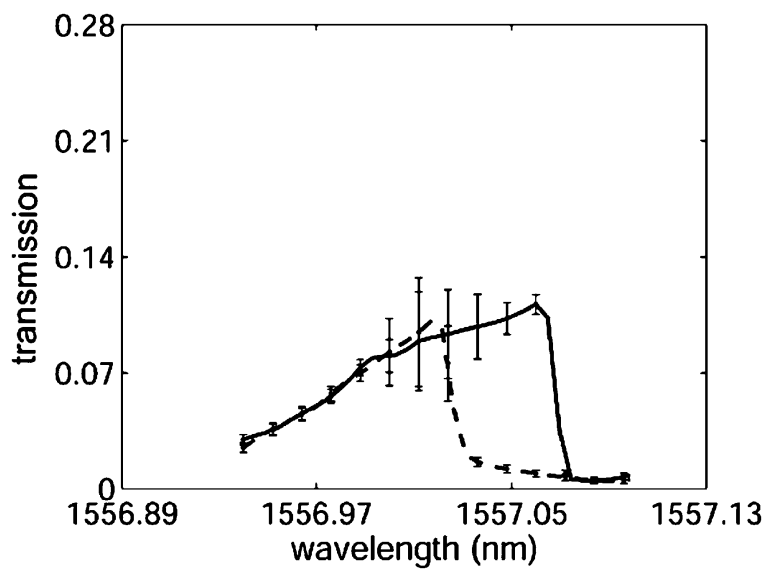

Fig. 16. Normalized average transmission and standard deviation at the drop port for an input power of $0.76 \mathrm{~mW}$.
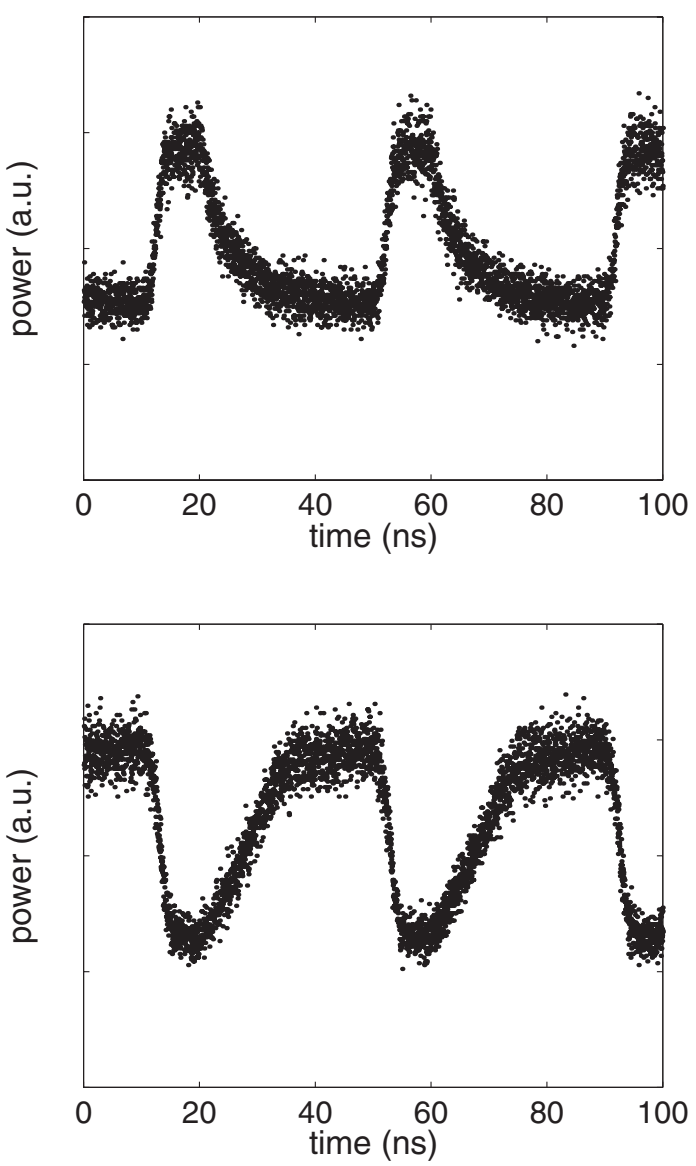

Fig. 17. Measured probe signals at the shorter (a) and the longer (b) wavelength side of the resonance for a peak pump power of $0.66 \mathrm{~mW}$. On the lower wavelength side, the converted data streams corrresponds to $10001000 \ldots$, while on the other side, $01110111 \ldots$ is obtained.

lower wavelength side of the probe resonance, while on the other side a $01110111 \ldots$ data pattern is created. While the rise time is typically fast, the maximum bitrate is clearly limited by the carrier recombination time, which is about $10 \mathrm{~ns}$. We measured an extinction ratio of respectively 2.7 and $3.8 \mathrm{~dB}$. At higher input power, we again observed unstable behaviour due to competition between thermal and free carrier refractive index contributions. This is shown 

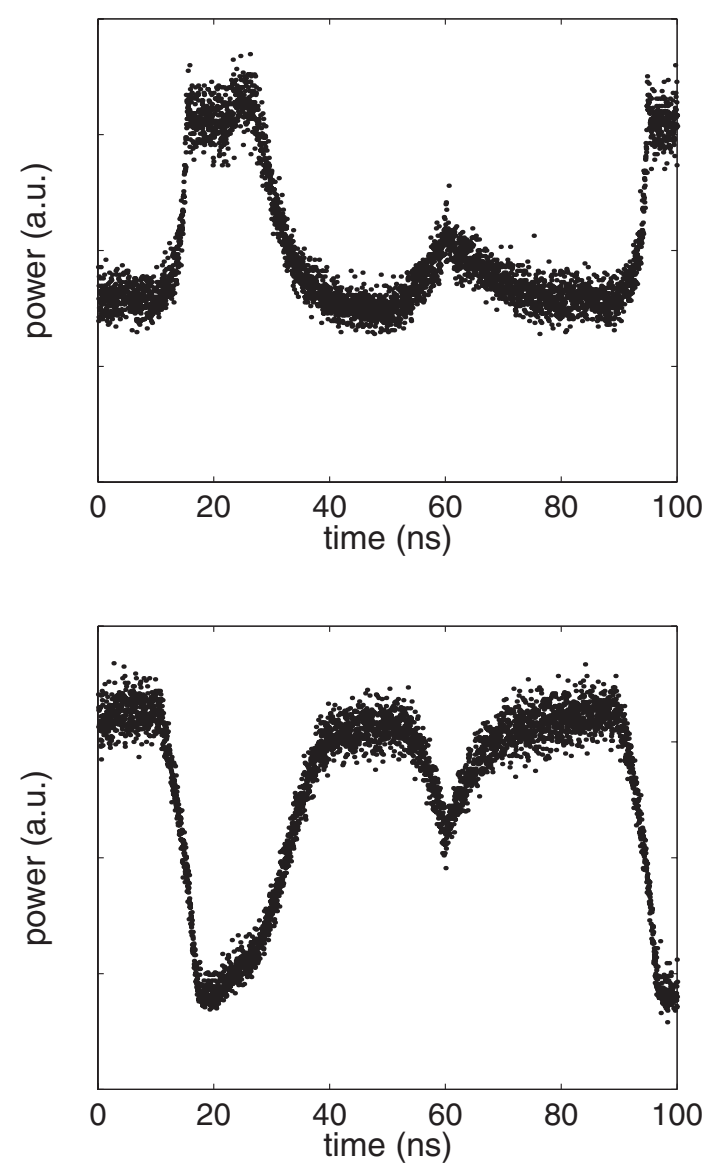

Fig. 18. Measured probe signals at the shorter (a) and the longer (b) wavelength side of the resonance for a peak pump power of $1.32 \mathrm{~mW}$.

in Fig. 18 for the same setup with a peak input power of $1.32 \mathrm{~mW}$. Significant distortions in the converted data pattern can be seen.

\section{Conclusions}

We have demonstrated a variety of structures and functions in SOI wires fabricated with CMOS based processes, including $248 \mathrm{~nm}$ deep UV lithography. Basic photonic circuit elements have losses and crosstalk low enough for basic functionality. However, for implementation in large and complex circuits, their performance will still need to be enhanced. We demonstrated single ring resonator channel drop filters with large drop efficiency and low adddrop crosstalk, lattice filters with low crosstalk and WDM channel spacings, and arrayed waveguide grating router devices with low insertion loss and crosstalk down to $-20 \mathrm{~dB}$ with WDM channel spacings. In plain photonic wires, we have shown $1.9 \mathrm{ps}$ nonlinear switching at low energy, an all-optical NOR gate and nonlinear self-distortion of picosecond optical pulses. Furthermore, we studied the reduction of the power and size requirements of nonlinear functions through the use of resonators.

With promising linear circuits and functions such as wavelength filters, and nonlinear functions for ultra-fast signal processing and switching being developed, photonic wire based photonic integrated circuits in silicon-on-insulator have great potential, especially combined with CMOS based technology.

\section{Acknowledgements}

The work was supported by the EU through the ISTePIXnet network of excellence and the IST-PICMOS project. P. Dumon thanks the Institute for the Promotion of Innovation through Science and Technology in Flanders (IWT-Vlaanderen) for a scholarship. G. Priem acknowledges the Flemish Fund for Scientific Research (FWO-Vlaanderen) for a doctoral research grant. W. Bogaerts and P. Bienstman acknowledge the FWO-Vlaanderen for a postdoctoral fellowship.

1) V. R. Almeida, C. A. Barrios, R. R. Panepucci and M. Lipson: Nature 431 (2004) 1081.

2) W. N. Ye, D. X. Xu, S. Janz, P. Cheben, M. J. Picard, B. Lamontagne and N. G. Tarr: J. Lightwave Technol. 23 (2005) 1308.

3) E. D. Palik: Handbook of Optical Constants of Solids (Academic Press, 1985).

4) M. Borselli, T. J. Johnson and O. Painter: Opt. Express 13 (2005) 1515 .

5) W. Bogaerts, R. Baets, P. Dumon, V. Wiaux, S. Beckx, D. Taillaert, B. Luyssaert, J. Van Campenhout, P. Bienstman and D. Van Thourhout: J. Lightwave Technol. 23 (2005) 401.

6) Y. A. Vlasov and S. J. McNab: Opt. Express 12 (2004) 1622.

7) T. Barwicz and H. A. Haus: J. Lightwave Technol. 23 (2005) 2719.

8) P. Dumon, G. Roelkens, W. Bogaerts, D. Van Thourhout, J. Wouters, S. Beckx, P. Jaenen and R. Baets: Group IV Photonics 2005, Antwerp, Belgium, 2005, p. 189.

9) A. Sakai, T. Fukazawa and T. Baba: J. Lightwave Technol. 22 (2004) 520.

10) T. Tsuchizawa, K. Yamada, H. Fukuda, T. Watanabe, J. Takahashi, M. Takahashi, T. Shoji, E. Tamechika, S. Itabashi and H. Morita: IEEE J. Sel. Top. Quantum Electron. 11 (2005) 232.

11) J. A. McCaulley, V. M. Donnelly, M. Vernon and I. Taha: Phys. Rev. B 49 (1994) 7408.

12) T. Baehr-Jones, M. Hochberg, C. Walker, E. Chan, D. Koshinz, W. Krug and A. Scherer: J. Lightwave Technol. 23 (2005) 4215.

13) A. Sakai, G. Hara and T. Baba: Jpn. J. Appl. Phys. 40 (2001) L383.

14) R. L. Espinola, M. C. Tsai, J. T. Yardley and R. M. Osgood: IEEE Photonics Technol. Lett. 15 (2003) 1366.

15) T. Chu, H. Yamada, S. Ishida and Y. Arakawa: Opt. Express 13 (2005) 10109.

16) R. Claps, D. Dimitropoulos, V. Raghunathan, Y. Han and B. Jalali: Opt. Express 11 (2003) 1731.

17) M. Sheik-Bahae, J. Wang and E. Van Stryland: IEEE J. Quantum Electron. 30 (1994) 249.

18) M. Dinu, F. Quochi and H. Garcia: Appl. Phys. Lett. 82 (2003) 2954.

19) K. DeLong, K. Rochford and G. Stegeman: Appl. Phys. Lett. 55 (1989) 1823.

20) G. Priem, P. Dumon, W. Bogaerts, D. Van Thourhout, G. Morthier and R. Baets: Opt. Express 13 (2005) 9623.

21) S. Preble, Q. Xu, B. Schmidt and M. Lipson: Opt. Lett. 30 (2005) 2891.

22) H. Tsang, C. Wong, T. Liang, I. Day, S. Robins, A. Harpin, J. Drake and M. Asghari: Appl. Phys. Lett. 80 (2002) 416.

23) G. Rieger, K. Virk and J. Young: Appl. Phys. Lett. 84 (2004) 900.

24) R. Orobtchouck, N. Schell, T. Benyattou and J. M. Fedeli: ECIO 2005, Grenoble, France, 2005.

25) D. K. Sparacin, S. J. Spector and L. C. Kimerling: J. Lightwave Technol. 23 (2005) 2455.

26) K. K. Lee, D. R. Lim, L. C. Kimerling, J. Shin and F. Cerrina: Opt. Lett. 26 (2001) 1888.

27) V. R. Almeida, R. R. Panepucci and M. Lipson: Opt. Lett. 28 (2003) 1302.

28) G. Roelkens, P. Dumon, W. Bogaerts, D. Van Thourhout and R. Baets: IEEE Photonics Technol. Lett. 17 (2005) 2613.

29) D. Taillaert, P. Bienstman and R. Baets: Opt. Lett. 29 (2004) 2749.

30) P. Dumon, W. Bogaerts, D. Van Thourhout, D. Taillaert, R. Baets, J. Wouters, S. Beckx and P. Jaenen: Opt. Express 14 (2006) 664. 
31) D. Taillaert, H. Chong, P. I. Borel, L. H. Frandsen, R. M. De La Rue and R. Baets: IEEE Photonics Technol. Lett. 15 (2003) 1249.

32) T. Fukazawa, T. Hirano, F. Ohno and T. Baba: Jpn. J. Appl. Phys. 43 (2004) 646.

33) S. G. Johnson, C. Manolatou, S. H. Fan, P. R. Villeneuve, J. D Joannopoulos and H. A. Haus: Opt. Lett. 23 (1998) 1855.

34) H. Yamada, T. Chu, S. Ishida and Y. Arakawa: IEEE Photonics Technol. Lett. 17 (2005) 585.

35) A. Sakai, T. Fukazawa and T. Baba: IEICE Trans. Electron. E85-C (2002) 1033.

36) T. Fukazawa, A. Sakai and T. Baba: Jpn. J. Appl. Phys. 41 (2002) L1461.

37) H. Yamada, T. Chu, S. Ishida and Y. Arakawa: Appl. Phys. Lett. 86 (2005) 191107.

38) M. Gnan, H. Chong and R. M. De La Rue: ECIO 2005, Grenoble, France, 2005, p. 310

39) J. S. Foresi, P. R. Villeneuve, J. Ferrera, E. R. Thoen, G. Steinmeyer, S. Fan, J. D. Joannopoulos, L. C. Kimmerling, H. I. Smith and E. P. Ippen: Nature 390 (1997) 143.

40) A. Kazmierczak, M. Briere, E. Drouard, P. Bontoux, P. Rojo-Romeo, I. O'Connor, X. Letartre, F. Gaffiot, R. Orobtchouk and T. Benyattou: IEEE Photonics Technol. Lett. 17 (2005) 1447.

$41)$ F. Ohno, T. Fukazawa and T. Baba: Jpn. J. Appl. Phys. 44 (2005) 5322.

42) K. Jinguji and M. Kawachi: J. Lightwave Technol. 13 (1995) 73.

43) P. Dumon, W. Bogaerts, D. Van Thourhout, D. Taillaert, V. Wiaux, S. Beckx, J. Wouters and R. Baets: ECOC 2004, Stockholm, Sweden, September 2004, Th. (post-deadline), p. 24.

44) K. Yamada, H. Fukuda, T. Watanabe, T. Tsuchizawa, T. Shoji and S. Itabashi: Group IV Photonics 2005, Antwerp, Belgium, 2005, p. FC1.

45) P. Dumon, W. Bogaerts, D. Van Thourhout, D. Taillaert, V. Wiaux, S. Beckx, J. Wouters and R. Baets: Group IV Photonics 2004, Hong Kong, 2004.

46) T. Fukazawa, F. Ohno and T. Baba: Jpn. J. Appl. Phys. 43 (2004) L673.

47) K. Sasaki, F. Ohno, A. Motegi and T. Baba: Electron. Lett. 41 (2005) 801 .

48) B. E. Little, J. S. Foresi, G. Steinmeyer, E. R. Thoen, S. T. Chu, H. A Haus, E. P. Ippen, L. C. Kimerling and W. Greene: IEEE Photonics Technol. Let. 10 (1998) 549.

49) P. Dumon, W. Bogaerts, V. Wiaux, J. Wouters, S. Beckx, J. Van Campenhout, D. Taillaert, B. Luyssaert, P. Bienstman, D. Van Thourhout and R. Baets: IEEE Photonics Technol. Lett. 16 (2004)
1328.

50) M. K. Chin and S. T. Ho: J. Lightwave Technol. 16 (1998) 1433.

51) P. Dumon, I. Christiaens, W. Bogaerts, V. Wiaux, J. Wouters, S. Beckx, D. Van Thourhout and R. Baets: 12th European Conf. on Integrated Optics (ECIO), Grenoble, France, April 2005, p. 196.

52) A. Vorckel, M. Monster, W. Henschel, P. H. Bolivar and H. Kurz: IEEE Photonics Technol. Lett. 15 (2003) 921.

53) J. Niehusmann, A. Vorckel, P. H. Bolivar, T. Wahbink, W. Henschel and H. Kurz: Opt. Lett. 29 (2004) 2861

54) T. Baehr-Jones, M. Hochberg, C. Walker and A. Scherer: Appl. Phys. Lett. 85 (2004) 3346.

55) R. L. Espinola, J. I. Dadap, R. M. Osgood, S. J. McNab and Y. A. Vlasov: Opt. Express 12 (2004) 3713.

56) Q. F. Xu, V. R. Almeida and M. Lipson: Opt. Lett. 30 (2005) 35.

57) R. L. Espinola, J. I. Dadap, R. M. Osgood, S. J. McNab and Y. A. Vlasov: Opt. Express 13 (2005) 4341.

58) H. Fukuda, K. Yamada, T. Shoji, M. Takahashi, T. Tsuchizawa, T. Watanabe, J. Takahashi and S. Itabashi: Opt. Express 13 (2005) 4629.

59) H. Yamada, M. Shirane, T. Chu, H. Yokoyama, S. Ishida and Y. Arakawa: Jpn. J. Appl. Phys. 44 (2005) 6541.

60) T. K. Liang, L. R. Nunes, T. Sakamoto, K. Sasagawa, T. Kawanishi, M. Tsuchiya, G. R. A. Priem, D. Van Thourhout, P. Dumon, R. Baets and H. K. Tsang: Opt. Express 13 (2005) 7298.

61) L. R. Nunes, T. K. Liang, K. S. Abedin, D. Van Thourhout, P. Dumon, R. Baets, H. K. Tsang, T. Miyazaki and M. Tsuchiya: ECOC 2005, Glasgow, September 2005, PD Th.4.2.3.

62) T. K. Liang, L. R. Nunes, M. Tsuchiya, K. S. Abedin, T. Miyazaki, D. Van Thourhout, P. Dumon, R. Baets and H. K. Tsang: OFC 2006, Anaheim, CA, March 2006, OFP1.

63) T. K. Liang, L. R. Nunes, M. Tsuchiya, K. S. Abedin, T. Miyazaki, D. Van Thourhout, P. Dumon, R. Baets and H. K. Tsang: submitted to CLEO 2006, May 2006.

64) I. Day, I. Evans, A. Knigths, F. Hopper, S. Roberts, J. Johnston, S. Day, J. Luff, H. Tsang and M. Asghari: OFC 2003, Vol. 1, p. 249.

$65)$ D. J. Moss, L. Fu, I. Littler and B. J. Eggleton: Electron. Lett. 41 (2005) 320 .

66) H. Fukuda, K. Yamada, T. Tsuchizawa, T. Watanabe, M. Takahashi and S. Itabashi: CLEO 2005, CWE1-4, p. 483.

67) G. Priem, I. Notebaert, B. Maes, P. Bienstman, G. Morthier and R. Baets: IEEE J. Sel. Top. Quantum Electron. 10 (2004) 1070.

68) V. Almeida and M. Lipson: Opt. Lett. 29 (2004) 2387.

69) H. Gibbs: Optical Bistability: Controlling Light with Light (Academic, Orlando, 1985). 